\title{
COMPENSATED CONVEXITY AND HAUSDORFF STABLE EXTRACTION OF INTERSECTIONS FOR SMOOTH MANIFOLDS
}

\author{
KEWEI ZHANG \\ School of Mathematical Sciences, University of Nottingham, University Park, \\ Nottingham, NG7 2RD, UK \\ kewei.zhang@nottingham.ac.uk \\ ANTONIO ORLANDO \\ Inst. de Estructuras \& Dept. de Mecánica, Universidad Nacional de Tucumán \\ Av. Independencia 1800, San Miguel de Tucumán, Argentina \\ aorlando@herrera.unt.edu.ar \\ ELAINE CROOKS \\ Department of Mathematics, Swansea University \\ Singleton Park, Swansea, SA2 8PP, UK \\ e.c.m.crooks@swansea.ac.uk \\ Received (Day Month Year) \\ Revised (Day Month Year) \\ Communicated by (xxxxxxxxxx)
}

\begin{abstract}
We apply compensated convex transforms to define a multiscale Hausdorff stable method to extract intersections between smooth compact manifolds represented by their characteristic functions or as point clouds embedded in $\mathbb{R}^{n}$. We prove extraction results on intersections of smooth compact manifolds and for points of high curvature. As a result of the Hausdorff-Lipschitz continuity of our transforms, we show that our method is stable against dense sampling of smooth manifolds with noise. Examples of explicitly calculated prototype models for some simple cases are presented, which are also used in the proofs of our main results. Numerical experiments in two and three-dimensional space, and applications to geometric objects are also shown.

Keywords: Compensated convex transforms; mathematical morphology; non-flat morphological operators; characteristic function; point clouds; Hausdorff-Lipschitz continuity; locality property; surface-to-surface intersection; transversal intersections; random samples.
\end{abstract}

AMS Subject Classification: 52A41, 49J52, 62H35, 14J17, 58K25, 53-XX

\section{Introduction}

In this paper we apply compensated convex transforms ${ }^{25,26,27}$ to define a Hausdorff stable multiscale method for extracting transversal intersections between smooth compact manifolds embedded in $\mathbb{R}^{n}$ represented by characteristic functions or by 
point clouds. If we denote by $M \subset \mathbb{R}^{n}$ the union of finitely many smooth compact manifolds $M_{k}$, for $k=1, \ldots, m$, we are interested in extracting the set of intersection points among the manifolds $M_{k}$. In $\mathbb{R}^{3}$ this is exactly the surface-tosurface and surface-to-curve intersection problem which has been studied extensively in computer-aided geometric design under the general terminology of shape interrogation. ${ }^{19}$

Finding the intersection of smooth compact manifolds is an important field not only for applications in engineering but also as a geometrical problem by itself. The traditional approach to surface-to-surface intersection problems is to consider parameterized polynomial surfaces and to solve systems of algebraic equations numerically based on real algebraic geometry. ${ }^{19}$ The application of these methods typically requires some topological information such as triangle mesh connectivity or a parameterization of the geometrical objects, hence they are difficult to implement in the case of free-form surfaces and of manifolds represented, for instance, by point clouds. For the latter case, other types of approaches are used. Such methods aim at identifying, according to some criteria, the points that are likely to belong to a neighborhood of the sharp feature. In Ref. 24 the criterion is based around the definition of a Gauss map clustering on local neighborhoods, which is then followed by a selective iterative process based on some sensitivity parameters; in Ref. 10 the borders of the various surface patches are extracted using a first order segmentation that identifies the candidate feature points that are subsequently processed as a graph, whereas in Ref. 9 the candidate points are selected using local normal estimates, local principle component analysis and tensor voting. All the results in these works are mostly justified by numerical experiments, and their stability properties, under dense sampling of the set $M$, are not known.

In this paper we are concerned with problems of geometric intersections between geometric objects of possibly different dimensions that can be either continuous or point-sampled. Using the compensated convex transforms, we will define the intersection filter $I_{\lambda}(\cdot, M)$ of scale parameter $\lambda>0$ and show that it is able to extract mainly two types of geometric singularities:

(i) Non-tangential surface-to-surface intersections under the transversal condition, and

(ii) Boundary points shared by two smooth manifolds.

Let $f: \mathbb{R}^{n} \mapsto \mathbb{R}$ be a bounded function, we recall that the quadratic lower and upper compensated convex transform ${ }^{25}$ (lower and upper transform for short) for a given $\lambda>0$ are defined for $x \in \mathbb{R}^{n}$ by

$$
C_{\lambda}^{l}(f)(x)=\operatorname{co}\left[\lambda|\cdot|^{2}+f\right](x)-\lambda|x|^{2} ; \quad C_{\lambda}^{u}(f)(x)=\lambda|x|^{2}-\operatorname{co}\left[\lambda|\cdot|^{2}-f\right](x)
$$

with $|x|$ the Euclidean norm of $x \in \mathbb{R}^{n}$ and co $[g]$ the convex envelope ${ }^{23,11}$ of a function $g: \mathbb{R}^{n} \mapsto \mathbb{R}$ bounded from below. 
From (1.1), it also follows that ${ }^{\mathrm{a}} C_{\lambda}^{l}(f)(x)$ is the envelope of all the quadratic functions with fixed quadratic term $\lambda|x|^{2}$ that are less than or equal to $f$, that is, $C_{\lambda}^{l}(f)(x)=\sup \left\{-\lambda|x|^{2}+\ell(x):-\lambda|y|^{2}+\ell(y) \leq f(y)\right.$ for all $y \in \mathbb{R}^{n}$ and $\ell$ affine $\}$,

whereas $C_{\lambda}^{u}(f)(x)$ is the envelope of all the quadratic functions with fixed quadratic term $\lambda|x|^{2}$ that are greater than or equal to $f$, that is,

$C_{\lambda}^{u}(f)(x)=\inf \left\{\lambda|x|^{2}+\ell(x): f(y) \leq \lambda|y|^{2}+\ell(y)\right.$ for all $y \in \mathbb{R}^{n}$ and $\ell$ affine $\}$.

The extraction filter $I_{\lambda}(\cdot ; M)$ is then defined as follows.

Definition 1.1. Let $K \subset \mathbb{R}^{n}$ be a non-empty compact set. We define the intersection extraction transform of scale $\lambda>0$ by

$$
I_{\lambda}(x ; K)=\left|C_{4 \lambda}^{u}\left(\chi_{K}\right)(x)-2\left(C_{\lambda}^{u}\left(\chi_{K}\right)(x)-C_{\lambda}^{l}\left(C_{\lambda}^{u}\left(\chi_{K}\right)\right)(x)\right)\right|, \quad x \in \mathbb{R}^{n} .
$$

By recalling from Ref. 27 the definition of the stable ridge transform of scale $\lambda$ and $\tau$ for the characteristic function $\chi_{K}$, with $K \subset \mathbb{R}^{n}$ compact set,

$$
S R_{\tau, \lambda}\left(\chi_{K}\right)=C_{\lambda}^{u}\left(\chi_{K}\right)-C_{\tau}^{l}\left(C_{\lambda}^{u}\left(\chi_{K}\right)\right),
$$

we can then express $I_{\lambda}(x ; K)$ in terms of $S R_{\lambda, \tau}\left(\chi_{K}\right)(x)$ for $\tau=\lambda$ as

$$
I_{\lambda}(x ; K)=\left|C_{4 \lambda}^{u}\left(\chi_{K}\right)(x)-2 S R_{\lambda, \lambda}\left(\chi_{K}\right)(x)\right|, \quad x \in \mathbb{R}^{n},
$$

which will be used to prove the Hausdorff-Lipschitz continuity of $I_{\lambda}(\cdot ; K)$.

The definition of the transform $I_{\lambda}(\cdot, K)$ is motivated by the following example which shows that $I_{\lambda}(\cdot ; K)$ can be used to remove or filter out 'regular points'.

Example 1.2. Consider the characteristic function $\chi_{\{0\}}(x)$ of the single point set $\{0\} \subset \mathbb{R}$. The following expressions of the compensated convex transforms are easy to calculate for $\lambda>0$ and $\tau>0$.

$$
\begin{aligned}
& C_{\lambda}^{u}\left(\chi_{\{0\}}\right)(x)= \begin{cases}\lambda(1 / \sqrt{\lambda}-|x|)^{2}, & |x| \leq 1 / \sqrt{\lambda}, \\
0, & |x| \geq 1 / \sqrt{\lambda}\end{cases} \\
& C_{\tau}^{l}\left(C_{\lambda}^{u}\left(\chi_{\{0\}}\right)\right)(x)= \begin{cases}\frac{\tau}{\lambda+\tau}-\tau x^{2}, & |x| \leq \frac{\sqrt{\lambda}}{\lambda+\tau} \\
C_{\lambda}^{u}\left(\chi_{\{0\}}\right)(x), & |x| \geq \frac{\sqrt{\lambda}}{\lambda+\tau} .\end{cases}
\end{aligned}
$$

Thus

$$
C_{\lambda}^{u}\left(\chi_{\{0\}}\right)(x)-C_{\tau}^{l}\left(C_{\lambda}^{u}\left(\chi_{\{0\}}\right)\right)(x)=\frac{\lambda}{\lambda+\tau} C_{(\lambda+\tau)^{2} / \lambda}^{u}\left(\chi_{\{0\}}\right)(x)
$$

${ }^{a}$ We are grateful to an anonymous referee of an earlier version of this manuscript for pointing out this characterization of the compensated convex transforms. 
so that

$$
C_{(\lambda+\tau)^{2} / \lambda}^{u}\left(\chi_{\{0\}}\right)(x)-\frac{\lambda+\tau}{\lambda}\left(C_{\lambda}^{u}\left(\chi_{\{0\}}\right)(x)-C_{\tau}^{l}\left(C_{\lambda}^{u}\left(\chi_{\{0\}}\right)\right)\right)(x)=0
$$

for all $x \in \mathbb{R}$. Therefore if we consider the special case $\lambda=\tau$, we obtain

$$
C_{4 \lambda}^{u}\left(\chi_{\{0\}}\right)(x)-2\left(C_{\lambda}^{u}\left(\chi_{\{0\}}\right)(x)-C_{\lambda}^{l}\left(C_{\lambda}^{u}\left(\chi_{\{0\}}\right)\right)(x)\right)=0
$$

that is, $I_{\lambda}(x ;\{0\})=0$ for all $x \in \mathbb{R}$.

While Example 1.2 can be generalised in a straightforward manner to characteristic functions of a single point or a single straight line in $\mathbb{R}^{2}$, we will further generalise this example to 'regular directions' and 'regular points' on manifolds $K$ and verify that at these points $I_{\lambda}(x ; K)=0$. This will permit, therefore, one to filter out the 'regular points' of the manifold and define the set of points $x$ such that $I_{\lambda}(x ; K)>0$ as intersection points and high curvature points of scale $\lambda$. For transversal intersection points of smooth manifolds, we will indeed prove that $I_{\lambda}(x ; K)>0$.

After this brief description, for the method we propose we claim the following:

(i) The intersection filter $I_{\lambda}(\cdot ; K)$ does not require any knowledge of tangential or normal directions of the underlying manifold, nor does its evaluation need any neighbourhood search even for point-sampled manifold;

(ii) The intersection filter $I_{\lambda}(\cdot ; K)$ is Hausdorff stable (see Theorem 3.1 below), so that for data sets under dense sampling and with small noise perturbation, it is still possible to extract intersection points;

(iii) The evaluation of $I_{\lambda}(\cdot ; K)$ relies on blind convexity based operations which are, in fact, local and do not involve heavy logical operations;

(iv) There is a rigorous mathematical theory underpinning our method;

$(v)$ We have explicitly calculated prototype models that justify and verify our method;

(vi) There are fast numerical schemes which allows the implementation of $I_{\lambda}(\cdot ; K)$.

As a conclusion of this introduction and to shed further light to the method we propose, it is useful to review the definition of $I_{\lambda}(\cdot ; K)$ within the context of morphological filtering. ${ }^{21}$ This is possible because compared with the basic operations in mathematical morphology, ${ }^{20,22}$ the lower and upper transforms can be viewed as 'one-step' morphological opening and closing, respectively. ${ }^{27}$ They, in fact, coincide with the critical mixed Moreau envelopes, that is,

$$
C_{\lambda}^{l}(f)(x)=M^{\lambda}\left(M_{\lambda}(f)\right)(x) \quad \text { and } \quad C_{\lambda}^{u}(f)(x)=M_{\lambda}\left(M^{\lambda}(f)\right)(x),
$$

where

$$
\begin{aligned}
& M_{\lambda}(f)(x)=\inf \left\{f(y)+\lambda|y-x|^{2}, y \in \mathbb{R}^{n}\right\} \quad \text { and } \\
& M^{\lambda}(f)(x)=\sup \left\{f(y)-\lambda|y-x|^{2}, y \in \mathbb{R}^{n}\right\}
\end{aligned}
$$


are the lower and upper Moreau envelopes, ${ }^{17,18,15,2,8}$ respectively. If we denote by $b_{\lambda}(x)=-\lambda|x|^{2}$ the quadratic structuring function, introduced for the first time in Refs. $12,4,13$, then with the notation of Refs. 20, 22, we have

$$
\begin{aligned}
& M_{\lambda}(f)(x)=\inf _{y \in \mathbb{R}^{n}}\left\{f(y)-b_{\lambda}(y-x)\right\}=: f \ominus b_{\lambda}, \\
& M^{\lambda}(f)(x)=\sup _{y \in \mathbb{R}^{n}}\left\{f(y)+b_{\lambda}(y-x)\right\}=: f \oplus b_{\lambda}
\end{aligned}
$$

that is, the Moreau lower and upper envelopes can be viewed as greyscale erosion and dilation with quadratic structuring function, respectively. ${ }^{16}$ Compared with (1.7) we have therefore

$$
C_{\lambda}^{l}(f)=\left(f \ominus b_{\lambda}\right) \oplus b_{\lambda} \quad \text { and } \quad C_{\lambda}^{u}(f)=\left(f \oplus b_{\lambda}\right) \ominus b_{\lambda},
$$

hence, by accounting for the definition $(1.4)$ of $I_{\lambda}(\cdot ; K)$, it follows

$I_{\lambda}(\cdot ; K)=\left|\left(\chi_{K} \oplus b_{4 \lambda}\right) \ominus b_{4 \lambda}-2\left(\left(\chi_{K} \oplus b_{\lambda}\right) \ominus b_{\lambda}-\left(\left(\left(\left(\chi_{K} \oplus b_{\lambda}\right) \ominus b_{\lambda}\right) \ominus b_{\lambda}\right) \oplus b_{\lambda}\right)\right)\right|$.

Given such an interpretation for $I_{\lambda}(\cdot ; K)$, the properties of $I_{\lambda}(\cdot ; K)$ could therefore be also analysed with the tools of the theory of morphological filtering. ${ }^{21}$

We finally observe that since lower and upper compensated convex transforms are also parameterized semiconvex and semiconcave envelopes of the function $f$, respectively, in the viscosity sense ${ }^{7}$ the Hessians of $C_{\lambda}^{l}(f(x))$ and $C_{\lambda}^{u}(f(x))$ satisfy

$$
y^{T} D^{2} C_{\lambda}^{l}(f)(x) \cdot y \geq-2|y|^{2} \lambda, \quad y^{T} D^{2} C_{\lambda}^{u}(f)(x) \cdot y \leq 2 \lambda|y|^{2}
$$

for $y \in \mathbb{R}^{n}$ and · denoting the inner product in $\mathbb{R}^{n}$. As a result, $C_{\lambda}^{l}(f)$ has a finite negative curvature lower bound and $C_{\lambda}^{u}(f)$ has a finite positive curvature upper bound. We may, therefore, view the compensated convex transforms also as curvature based one-step morphological opening and closing.

An outline of the remaining parts of the paper is as follows. Section 2 contains some definitions and recalls results from convex analysis and the theory of compensated convex transforms as developed in Refs. 25, 27 which will be then used for the proof of the main results. These are given in Section 3, where we state the Hausdorff stability of the intersection filter $I_{\lambda}(\cdot ; K)$ and describe its behaviour at the 'regular' and singular points of piecewise smooth compact manifolds. Section 4 consists of two subsections. The first one contains some prototype models for which we are able to obtain analytical expressions for $I(\cdot ; K)$ which we use to verify the method we propose. The second one, very briefly, reports some numerical experiments based on a computer implementation of the filter $I_{\lambda}(\cdot ; K)$ for different types of compact sets $K$. The numerical examples highlight the effectiveness of the transform $I_{\lambda}(\cdot ; K)$ and its Hausdorff stability property against point samples. The proofs of the main results are detailed in the final Section 5. 


\section{Notation and Preliminaries}

This section presents a brief overview of some basic results in convex analysis and in the theory of compensated convex transforms that will be used in the sequel for the proof of the main results; for a comprehensive account of convex analysis, refer to Refs. 11, 23, and to Refs. 25, 27 for an account of the theory of compensated convex transforms.

Proposition 2.1. Let $f: \mathbb{R}^{n} \mapsto \mathbb{R}$ be coercive in the sense that $f(x) /|x| \rightarrow \infty$ as $|x| \rightarrow \infty$, and $x_{0} \in \mathbb{R}^{n}$. Then

(i) The value co $[f]\left(x_{0}\right)$ of the convex envelope of $f$ at $x_{0} \in \mathbb{R}^{n}$ is given by

$$
\begin{gathered}
\operatorname{co}[f]\left(x_{0}\right)=\inf _{i=1, \ldots, n+1}\left\{\sum_{i=1}^{n+1} \lambda_{i} f\left(x_{i}\right): \sum_{i=1}^{n+1} \lambda_{i}=1, \sum_{i=1}^{n+1} \lambda_{i} x_{i}=x_{0},\right. \\
\left.\lambda_{i} \geq 0, x_{i} \in \mathbb{R}^{n}\right\} .
\end{gathered}
$$

If, in addition, $f$ is lower semicontinuous, the infimum is reached by some $\left(\lambda_{i}^{*}, x_{i}^{*}\right)$ for $i=1,2, \ldots, n+1$ with $\left(x_{i}^{*}, f\left(x_{i}^{*}\right)\right)$ 's lying in the intersection of a supporting plane of the epigraph of $f$, epi $(f)$, and epi $(f)$.

(ii) The value co $[f]\left(x_{0}\right)$, for $f$ taking only finite values, can also be obtained as follows:

$$
\operatorname{co}[f]\left(x_{0}\right)=\sup \left\{\ell\left(x_{0}\right): \ell \text { affine and } \ell(y) \leq f(y) \text { for all } y \in \mathbb{R}^{n}\right\}
$$

with the sup attained by an affine function $\ell^{*} \in \operatorname{Aff}\left(\mathbb{R}^{n}\right)$.

We will also introduce the following local version of convex envelope at a point.

Definition 2.2. Let $r>0$ and $x_{0} \in \mathbb{R}^{n}$. Suppose $f: \bar{B}\left(x_{0} ; r\right) \mapsto \mathbb{R}$ is a bounded function in $\bar{B}\left(x_{0} ; r\right)$. Then the value $\operatorname{co}_{\bar{B}\left(x_{0} ; r\right)}[f]\left(x_{0}\right)$ of the local convex envelope of $f$ at $x_{0}$ in $\bar{B}\left(x_{0} ; r\right)$ is defined by

$$
\begin{array}{r}
\mathrm{co}_{\bar{B}\left(x_{0} ; r\right)}[f]\left(x_{0}\right)=\inf _{i=1, \ldots, n+1}\left\{\sum_{i=1}^{n+1} \lambda_{i} f\left(x_{i}\right): \sum_{i=1}^{n+1} \lambda_{i}=1, \sum_{i=1}^{n+1} \lambda_{i} x_{i}=x_{0},\right. \\
\left.\lambda_{i} \geq 0,\left|x_{i}-x_{0}\right| \leq r, x_{i} \in \mathbb{R}^{n}\right\} .
\end{array}
$$

Definition 2.3. Given a non-empty subset $E$ of $\mathbb{R}^{n}$ and $\delta>0$, we define the $\delta$-neighbourhood $E^{\delta}$ of $E$ by

$$
E^{\delta}=\left\{x \in \mathbb{R}^{n}: \operatorname{dist}(x ; E)<\delta\right\},
$$

where $\operatorname{dist}(x ; E)=\inf \{|x-y|, y \in E\}$.

Note that $E^{\delta}$ is an open subset of $\mathbb{R}^{n}$. 
Definition 2.4. Let $E, F$ be non-empty subsets of $\mathbb{R}^{n}$. The Hausdorff distance between $E$ and $F$ is defined in Ref. 1 by

$$
\operatorname{dist}_{\mathcal{H}}(E, F)=\inf \left\{\delta>0: F \subset E^{\delta} \text { and } E \subset F^{\delta}\right\} .
$$

We recall also the following ordering properties for compensated convex transforms which can be found in Ref. 25:

$$
C_{\lambda}^{l}(f)(x) \leq f(x) \leq C_{\lambda}^{u}(f)(x), \quad x \in \mathbb{R}^{n},
$$

whereas for $f \leq g$ in $\mathbb{R}^{n}$, we have that

$$
C_{\lambda}^{l}(f)(x) \leq C_{\lambda}^{l}(g)(x) \quad \text { and } \quad C_{\lambda}^{u}(f)(x) \leq C_{\lambda}^{u}(g)(x), \quad x \in \mathbb{R}^{n} .
$$

Proposition 2.5. (Translation invariance property) For any $f: \mathbb{R}^{n} \mapsto \mathbb{R}$ bounded below and for any affine function $\ell: \mathbb{R}^{n} \mapsto \mathbb{R}, \operatorname{co}[f+\ell]=\operatorname{co}[f]+\ell$. Consequently, both $C_{\lambda}^{u}(f)$ and $C_{\lambda}^{l}(f)$ are translation invariant against the weight function, that is

$$
\begin{aligned}
& C_{\lambda}^{l}(f)(x)=\operatorname{co}\left[\lambda\left|(\cdot)-x_{0}\right|^{2}+f\right](x)-\lambda\left|x-x_{0}\right|^{2}, \\
& C_{\lambda}^{u}(f)(x)=\lambda\left|x-x_{0}\right|^{2}-\operatorname{co}\left[\lambda\left|(\cdot)-x_{0}\right|^{2}-f\right](x)
\end{aligned}
$$

for all $x \in \mathbb{R}^{n}$ and for every fixed $x_{0}$. Hence, at $x_{0}$,

$$
C_{\lambda}^{l}(f)\left(x_{0}\right)=\operatorname{co}\left[\lambda\left|(\cdot)-x_{0}\right|^{2}+f\right]\left(x_{0}\right), \quad C_{\lambda}^{u}(f)\left(x_{0}\right)=-\operatorname{co}\left[\lambda\left|(\cdot)-x_{0}\right|^{2}-f\right]\left(x_{0}\right) .
$$

Fundamental is then for both theoretical and numerical developments, the following property on the locality of the compensated transforms (refer to Theorem 3.10 of Ref. 27).

Theorem 2.6. (The locality and density properties for bounded functions) Suppose $f: \mathbb{R}^{n} \mapsto \mathbb{R}$ is bounded, satisfying $|f(x)| \leq M$ for some $M>0$ and for all $x \in \mathbb{R}^{n}$. Let $\lambda>0$ and $x_{0} \in \mathbb{R}^{n}$. The following locality properties hold:

$$
\begin{aligned}
& C_{\lambda}^{l}(f)\left(x_{0}\right)=\operatorname{co}_{\bar{B}\left(x_{0} ; R_{\lambda, M}\right)}\left[f+\lambda\left|(\cdot)-x_{0}\right|^{2}\right]\left(x_{0}\right), \\
& C_{\lambda}^{u}(f)\left(x_{0}\right)=-\operatorname{co}_{\bar{B}\left(x_{0} ; R_{\lambda, M}\right)}\left[\lambda\left|(\cdot)-x_{0}\right|^{2}-f\right]\left(x_{0}\right)
\end{aligned}
$$

with $R_{\lambda, M} \leq 2 \sqrt{2} \sqrt{\frac{M}{\lambda}}$, and $\operatorname{co}_{\bar{B}\left(x_{0} ; R_{\lambda, M}\right)}[g]\left(x_{0}\right)$ the value of the local convex envelope of $g$ at $x_{0}$ in $\bar{B}\left(x_{0} ; R_{\lambda, M}\right)$ defined according to Definition 2.2.

Remark 2.7. From Lemma 3.9 of Ref. 27, the result of Theorem 2.6 applied to the characteristic function $\chi_{E}$ of a non-empty closed subset $E \subset \mathbb{R}^{n}$ specializes, for any $x_{0} \in \mathbb{R}^{n}$, as

$$
C_{\lambda}^{u}\left(\chi_{E}\right)\left(x_{0}\right)=-\operatorname{co}_{\bar{B}\left(x_{0} ; R_{\lambda}\right)}\left[\lambda\left|(\cdot)-x_{0}\right|^{2}-\chi_{E}\right]\left(x_{0}\right),
$$

with $\operatorname{co}_{\bar{B}\left(x ; R_{\lambda}\right)}\left[\lambda\left|(\cdot)-x_{0}\right|^{2}-\chi_{E}\right]$ the convex envelope taken in the ball $\bar{B}\left(x_{0} ; R_{\lambda}\right)$ and the better estimate $R_{\lambda}=(1+\sqrt{2}) / \sqrt{\lambda}$. This result can then be written as

$$
C_{\lambda}^{u}\left(\chi_{E}\right)\left(x_{0}\right)=C_{\lambda}^{u}\left(\chi_{E \cap \bar{B}\left(x_{0} ; R_{\lambda}\right)}\right)\left(x_{0}\right)
$$

for $x_{0} \in \mathbb{R}^{n}$. 
We will need also the following characterization of the upper transform of the characteristic function of a non-empty subset of $\mathbb{R}^{n}$ which was established in Ref. 27 (see therein Theorem 3.4).

Theorem 2.8. (Expansion Theorem) Let $E \subset \mathbb{R}^{n}$ be a non-empty set and let $\lambda>0$ be fixed, then

$$
C_{\lambda}^{u}\left(\chi_{E}\right)(x) \quad \begin{cases}=1 & \text { if } x \in \bar{E} \\ =0 & \text { if } x \in\left(E^{1 / \sqrt{\lambda}}\right)^{c} \\ \in(0,1) & \text { if } x \in E^{1 / \sqrt{\lambda}} \backslash \bar{E} .\end{cases}
$$

The following propositions will enable us to extend some of our prototype examples to higher dimensional spaces.

Proposition 2.9. (Separation of variable property) For $x \in \mathbb{R}^{n}, y \in \mathbb{R}^{m}$, let $f(x, y)=g(x)+h(y)$ with $g: \mathbb{R}^{n} \mapsto \mathbb{R}$ and $h: \mathbb{R}^{m} \mapsto \mathbb{R}$ both bounded below. Then $\mathrm{co}_{\mathbb{R}^{n+m}}[f]=\mathrm{co}_{\mathbb{R}^{n}}[g]+\mathrm{co}_{\mathbb{R}^{m}}[h]$, where $\mathrm{co}_{\mathbb{R}^{n+m}}[f]$, co $\mathbb{R}^{n}[g]$ and $\mathrm{co}_{\mathbb{R}^{m}}[h]$ are the convex envelopes of $f, g$ and $h$ in $\mathbb{R}^{n+m}, \mathbb{R}^{n}$ and $\mathbb{R}^{m}$, respectively. Hence, we have, for all $x \in \mathbb{R}^{n}$ and $y \in \mathbb{R}^{m}$,

$$
\begin{aligned}
& C_{\mathbb{R}^{n+m}, \lambda}^{l}(f)(x, y)=C_{\mathbb{R}^{n}, \lambda}^{l}(g)(x)+C_{\mathbb{R}^{m}, \lambda}^{l}(h)(y) ; \\
& C_{\mathbb{R}^{n+m}, \lambda}^{l}(f)(x, y)=C_{\mathbb{R}^{n}, \lambda}^{l}(g)(x)+C_{\mathbb{R}^{m}, \lambda}^{l}(h)(y) .
\end{aligned}
$$

Proposition 2.10. (Partial Rotation Invariance property) Let $f(x, y)$ be a bounded function for $(x, y) \in \mathbb{R}^{2}$ such that $f$ is even in $y \in \mathbb{R}$, that is, for all $(x, y) \in \mathbb{R}^{2}$, $f(x, y)=f(x,-y)$. Let $F: \mathbb{R} \times \mathbb{R}^{n} \mapsto \mathbb{R}$ be defined by $F(x, z)=f(x,|z|)$ for $x \in \mathbb{R}$ and $z \in \mathbb{R}^{n}$. Then

$$
\mathrm{CO}_{\mathbb{R}^{n+1}}[F](x, z)=\mathrm{co}_{\mathbb{R}^{2}}[f](x,|z|),
$$

where $\mathrm{co}_{\mathbb{R}^{n+1}}[F]$ and $\mathrm{co}_{\mathbb{R}^{2}}[f]$ are the convex envelopes of $F$ and $f$ in $\mathbb{R}^{n+1}$ and $\mathbb{R}^{2}$, respectively.

Since $I_{\lambda}(\cdot ; K)$ is defined in terms of the mixed compensated compact transform $C_{\lambda}^{l}\left(C_{\lambda}^{u}\left(\chi_{K}\right)\right)$, in the sequel to simplify calculations, it will be convenient to refer to the distance based function introduced in Ref. 27

$$
D_{\lambda}(x ; K):=\max \{0,1-\sqrt{\lambda} \operatorname{dist}(x ; K)\}, \quad x \in \mathbb{R}^{n},
$$

given that (see Proposition 5.3 of Ref. 27)

$$
C_{\lambda}^{u}\left(\chi_{K}\right)(x)=C_{\lambda}^{u}\left(D_{\lambda}^{2}(\cdot ; K)\right)(x), \quad x \in \mathbb{R}^{n} .
$$

Taking into account (2.9) and the ordering property of the compensated convex transforms (2.4), we can then conclude that

$$
C_{\lambda}^{u}\left(\chi_{K}\right)(x)=C_{\lambda}^{u}\left(D_{\lambda}^{2}(\cdot ; K)\right)(x) \geq D_{\lambda}^{2}(x ; K), \quad x \in \mathbb{R}^{n},
$$


and, using (2.5), that

$$
C_{\lambda}^{l}\left(C_{\lambda}^{u}\left(\chi_{K}\right)\right)(x) \geq C_{\lambda}^{l}\left(D_{\lambda}^{2}(\cdot ; K)\right)(x), \quad x \in \mathbb{R}^{n} .
$$

The next result will be useful in the construction of prototypes in $\mathbb{R}^{3}$; see Remark 4.2 and Figure 2.

Proposition 2.11. Suppose $m \geq 1$ and $n \geq 1$. Let $K_{m} \subset \mathbb{R}^{m}$ be a closed set and define the set $K_{m}^{n}=K_{m} \times \mathbb{R}^{n}$ and its subset $K_{m}^{0, n}=K_{m} \times\left\{0 \in \mathbb{R}^{n}\right\}$. The set $K_{m}^{n}$ is referred to as the lift of $K_{m}$ in the larger space $\mathbb{R}^{n+m}$ while $K_{m}^{0, n}$ is the embedding of $K_{m}$ into $\mathbb{R}^{n+m}$. We have then the following:

(i) For $K_{m}^{n}$, we have

$$
\begin{aligned}
& C_{\lambda}^{u}\left(\chi_{K_{m}^{n}}\right)(x, z)=C_{\lambda}^{u}\left(\chi_{K_{m}}\right)(x), \quad x \in \mathbb{R}^{m}, z \in \mathbb{R}^{n}, \\
& D_{\lambda}^{2}\left((x, z) ; K_{m}^{n}\right)=D_{\lambda}^{2}\left(x ; K_{m}\right), \quad x \in \mathbb{R}^{m}, z \in \mathbb{R}^{n},
\end{aligned}
$$

where the right hand sides of the equalities are defined in $\mathbb{R}^{m}$.

(ii) For $K_{m}^{0, n}$, we have

$$
\begin{aligned}
& C_{\lambda}^{u}\left(\chi_{K_{m}^{0, n}}\right)(x, 0)=C_{\lambda}^{u}\left(\chi_{K_{m}}\right)(x), \quad x \in \mathbb{R}^{m}, z \in \mathbb{R}^{n}, \\
& D_{\lambda}^{2}\left((x, 0) ; K_{m}^{0, n}\right)=D_{\lambda}^{2}\left(x ; K_{m}\right), \quad x \in \mathbb{R}^{m}, 0 \in \mathbb{R}^{n},
\end{aligned}
$$

where the right hand sides of the equalities are defined in $\mathbb{R}^{m}$.

The following results on the Hausdorff-Lipschitz continuity of $C_{\lambda}^{u}\left(\chi_{K}\right)$ and $S R_{\lambda}\left(\chi_{K}\right)$ were established in Ref. 27 (see therein Theorem 5.5 and Theorem 5.9, respectively) and will be used to prove the stability of the filter $I_{\lambda}(x ; K)$.

Theorem 2.12. (Hausdorff-Lipschitz continuity) Let E, F be non-empty compact subsets of $\mathbb{R}^{n}$. Assume $\lambda>0$. Then for $x \in \mathbb{R}^{n}$

$$
\begin{aligned}
& \left|C_{\lambda}^{u}\left(\chi_{E}\right)(x)-C_{\lambda}^{u}\left(\chi_{F}\right)(x)\right| \leq 2 \sqrt{\lambda} \operatorname{dist}_{\mathcal{H}}(E, F) ; \\
& \left|S R_{\lambda, \tau}\left(\chi_{E}\right)(x)-S R_{\lambda, \tau}\left(\chi_{F}\right)(x)\right| \leq 4 \sqrt{\lambda} \operatorname{dist}_{\mathcal{H}}(E, F) .
\end{aligned}
$$

We will also need a simple Hausdorff-Lipschitz continuity property for the lower transform of $D_{\lambda}^{2}(x ; K)$, of which a proof is given in Section 5 .

Lemma 2.13. Suppose $\lambda>0$ and let $E, F \subset \mathbb{R}^{n}$ be non-empty and compact. Then for $x \in \mathbb{R}^{n}$

$$
\left|C_{\lambda}^{l}\left(D_{\lambda}^{2}(\cdot ; E)\right)(x)-C_{\lambda}^{l}\left(D_{\lambda}^{2}(\cdot ; F)\right)(x)\right| \leq 2 \sqrt{\lambda} \operatorname{dist}_{\mathcal{H}}(E, F) .
$$

\section{Main Results}

The first result of this section is the Hausdorff stability of the intersection filter $I(\cdot ; K)$. This is clearly a desirable property especially when one deals with point samples of geometric objects. 
Theorem 3.1. (Hausdorff-Lipschitz continuity of the intersection transform) Let $E, F$ be non-empty compact subsets of $\mathbb{R}^{n}$. Assume $\lambda>0$. Then, there holds

$$
\left|I_{\lambda}(x ; E)-I_{\lambda}(x ; F)\right| \leq 12 \sqrt{\lambda} \operatorname{dist}_{\mathcal{H}}(E, F), \quad x \in \mathbb{R}^{n} .
$$

We proceed next to analyze the behaviour of $I(x ; K)$ for different types of points and directions. Our objective is to show that we can use $I(x ; K)$ to detect the geometric singularities of a manifold. We will therefore introduce first the notion of $\delta$-regular directions and $\delta$-regular points as follows:

Definition 3.2. Let $K \subset \mathbb{R}^{n}$ be non-empty and compact and let $x \in K$. Let $e \in \mathbb{R}^{n}$ be a unit vector. Then

(i) We say that $e$ is a $\delta$-regular direction of $x$ if $\bar{B}(x+\delta e ; \delta) \cap K=\bar{B}(x-$ $\delta e ; \delta) \cap K=\{x\}$.

(ii) Suppose $K$ is locally an $m$-dimensional $C^{1}$ manifold with $1 \leq m \leq n-1$ in a neighbourhood of $x$ with tangent subspace $T_{x}$ and normal subspace $N_{x}$. If every unit vector $e \in N_{x}$ is a $\delta$ regular direction of $x$, we say that $x$ is a $\delta$-regular point of $K$.

Remark 3.3. If $M \subset \mathbb{R}^{n}$ is a compact $C^{2}$ manifold without boundary, every point of $M$ is a $\delta$-regular point for some fixed $\delta>0$. The point 0 of Example 1.2 where $K=\{0\} \subset \mathbb{R}$, is also a $\delta$-regular point, for any $\delta>0$, according to Definition 3.2.

The definition of regular points as given above is justified by the following Theorem which characterizes the value of $I_{\lambda}(\cdot ; K)$ at such points.

Theorem 3.4. Suppose $K \subset \mathbb{R}^{n}$ is a non-empty compact set and e is a $\delta$-regular direction of $x \in K$, then $I_{\lambda}(y ; K)=0$ for $y \in[x-\delta e, x+\delta e]:=\{x+t \delta e,-1 \leq$ $t \leq 1\}$ when $\lambda \geq 1 / \delta^{2}$. In particular, we have that at the point $x$

$$
C_{\lambda}^{l}\left(C_{\lambda}^{u}\left(\chi_{K}\right)\right)(x)=1 / 2 .
$$

If $K$ is a $C^{1}$ manifold in a neighbourhood of $x \in K$ and $x$ is a $\delta$-regular point of $K$, then $I_{\lambda}(y ; K)=0$ if $y-x \in N_{x}$ and $|y-x| \leq \delta$.

\section{Remark 3.5.}

(a) From the proof of Theorem 3.4, it will follow that if $x \in K$ is a $\delta$-regular point for some $\delta>0, C_{\lambda}^{l}\left(C_{\lambda}^{u}\left(\chi_{K}\right)\right)(x)=1 / 2$ and $x$ is the maximum point in the interval $[x-\delta e, x+\delta]$ for every regular direction $e$. This means that we could also simply use the mixed transform $C_{\lambda}^{l}\left(C_{\lambda}^{u}\left(\chi_{K}\right)\right)(x)$ to define an intersection filter by taking the threshold above $1 / 2$. By contrast, the advantage of using $I_{\lambda}(\cdot ; K)$ is that the regular points will be removed by the transform itself, leaving only the singular ones. 
(b) Given the inequality (2.11), we could define also another intersection filter as

$$
J_{\lambda}(x ; K)=\left|C_{4 \lambda}^{u}\left(\chi_{K}\right)(x)-2\left(C_{\lambda}^{u}\left(\chi_{K}\right)(x)-C_{\lambda}^{l}\left(D_{\lambda}^{2}(\cdot ; K)\right)(x)\right)\right| .
$$

Although $J_{\lambda}(\cdot ; K)$ would be a weaker intersection filter than $I_{\lambda}(\cdot ; K)$, we will nevertheless later use $J_{\lambda}(\cdot ; K)$ to establish strong extractions of transversal intersections. By applying $J_{\lambda}(\cdot ; K)$, we will not be required to compute mixed compensated convex transforms. This will makes, therefore, our theoretical arguments much easier. From a practical point of view, however, since $D_{\lambda}^{2}(x ; K)$ is defined by the distance function, the computation of $J_{\lambda}(\cdot ; K)$ will depend on the accuracy of the numerical computation of $\left.D_{\lambda}^{2}(x ; K)\right)$. By contrast, if we use $I_{\lambda}(x ; K)$, the only information we need is the characteristic function $\chi_{K}$ without using any external functions whose numerical accuracy could be out of our control.

For compact $C^{2} m$-dimensional manifolds with $1 \leq m \leq n-1$, Theorem 3.4 implies that $I_{\lambda}(y ; K)=0$ for all $\delta$-regular points $y \in K$ when $\lambda>0$ is sufficiently large. This result motivates the following definition of singular points which can be extracted by $I_{\lambda}(\cdot ; K)$.

Definition 3.6. Let $K$ be a closed non-empty subset of $\mathbb{R}^{n}$. Assume $\lambda>0$. A point $x \in K$ is called a strongly extractable singular point of $K$ if there exists a constant $c_{x}>0$, depending at most only on $x$, such that $I_{\lambda}(x ; K) \geq c_{x}>0$ for sufficiently large $\lambda>0$.

The definition is justified by looking at the value of $I_{\lambda}(x ; M)$ for some types of geometric singular points of a manifold $M$. We will assume in the sequel, without loss of generality, that the geometric singularity is located at the point 0 of $\mathbb{R}^{n}$, and therefore that we are interested to know what we can say on the value of $I_{\lambda}(0 ; M)$ with $0 \in M \subset \mathbb{R}^{n}$. We will first consider the case that $M$ is a finite union of some subspaces of $\mathbb{R}^{n}$, that is, $L=\bigcup_{k=1}^{m} L_{k} \subset \mathbb{R}^{n}$ with $1 \leq \operatorname{dim}\left(L_{k}\right) \leq n-1$, under the assumptions that there are no subspaces which are contained in other subspaces and $\operatorname{span}\left[L_{i}, i=1, \ldots, m\right]=\mathbb{R}^{n}$. Subsequently, we will extend this result to general $C^{2}$ embedded manifolds in $\mathbb{R}^{n}$ with $n \geq 2$. Before proceeding, we will need first the following sufficient condition for $I_{\lambda}(x ; K)>0$.

Lemma 3.7. Let $K \subset \mathbb{R}^{n}$ be a closed non-empty set and $x \in K$. Assume $\lambda>0$. If $C_{\lambda}^{l}\left(D_{\lambda}^{2}(\cdot ; K)\right)(x)>1 / 2$ then $I_{\lambda}(x ; K)>0$.

Theorem 3.8. Suppose $n \geq 2$ and let $\left\{e_{1}, e_{2}, \ldots, e_{m}\right\} \subset \mathbb{R}^{n}$ be a finite collection of unit vectors. We define the ray $L_{i}^{+}=\left\{t e_{i}, t \geq 0\right\}$ for $i=1,2, \ldots, m$. Let $L=\bigcup_{i=1}^{m} L_{i}^{+} ;$clearly $0 \in L$. Then

$$
C_{\lambda}^{l}\left(D_{\lambda}^{2}(\cdot ; L)\right)(0)>1 / 2
$$


if and only if

$$
\operatorname{span}\left[e_{1}, e_{2}, \ldots, e_{m}\right]=\mathbb{R}^{n} .
$$

Furthermore, if (3.5) holds, then there exists a constant $\mu_{L}>0$ independent of $\lambda>0$ such that

$$
C_{\lambda}^{l}\left(D_{\lambda}^{2}(\cdot ; L)\right)(0)=1 / 2+\mu_{L}
$$

Consequently,

$$
I_{\lambda}(0 ; L) \geq 2 \mu_{L}>0,
$$

and the point 0 is a strongly extractable singular point of $L$.

The content of Theorem 3.8 can be generalized to the case where in place of the positive rays $L_{i}^{+}$originating at the point 0 , we have the range $\Gamma_{i}^{+}$of smooth curves originating at the same point 0 .

Proposition 3.9. Let $\gamma_{i}:[0, \delta] \mapsto \mathbb{R}^{n}$ for $i=1,2, \ldots, m$ be finitely many $C^{2}$ compact curves in $\mathbb{R}^{n}$ parameterized by arc-length with $m \geq n$ and denote by $\Gamma_{i}$ the range of the regular curves $\gamma_{i}$, i.e. $\Gamma_{i}=\left\{\gamma_{i}(s) \in \mathbb{R}^{n}, s \in[0, \delta]\right\}$ and by $\Gamma=\bigcup_{i=1}^{m} \Gamma_{i}$. Assume that $0 \in \bigcap_{i=1}^{m} \Gamma_{i}$ and that $\gamma_{i}(0)=0$ for $i=1,2, \ldots, m$. Suppose that the one-sided tangent vectors $e_{i}:=\gamma_{i,+}^{\prime}(0)=\lim _{s \rightarrow 0_{+}}\left(\gamma_{i}(s)-\gamma_{i}(0)\right) / s$ for $i=1,2, \ldots, m$ are such that $\operatorname{span}\left[\gamma_{1,+}^{\prime}(0), \gamma_{2,+}^{\prime}(0), \ldots, \gamma_{m,+}^{\prime}(0)\right]=\mathbb{R}^{n}$. Then for $\lambda>0$ sufficiently large, we have

$$
C_{\lambda}^{l}\left(D_{\lambda}^{2}(\cdot ; \Gamma)\right)(0) \geq 1 / 2+\mu_{L} / 2
$$

where the constant $\mu_{L}>0$ is independent of $\lambda>0$ and is such that $C_{\lambda}^{l}\left(D_{\lambda}^{2}(\cdot ; L)\right)(0)=1 / 2+\mu_{L}$ where $L=\bigcup_{i=1}^{n} L_{i}^{+}$with $L_{i}^{+}$the positive ray generated by the one-sided tangent vector $e_{i}:=\gamma_{i,+}^{\prime}(0)$, that is, $L_{i}^{+}=\left\{t \gamma_{i,+}^{\prime}(0), t \geq 0\right\}$. As a result, we have that

$$
I_{\lambda}(0 ; \Gamma) \geq \mu_{L}
$$

for $\lambda>0$ sufficiently large and the point 0 is a strongly extractable singular point of $\Gamma$.

As a direct consequence of Theorem 3.8 and Proposition 3.9, we can determine the behaviour of $I_{\lambda}(0 ; M)$ for the case where $M$ is the finite union of compact $C^{2}$ $m$-dimensional manifolds with $1 \leq m \leq n-1$ and $0 \in \cap_{j=1}^{k} M_{j}$.

Theorem 3.10. Let $M_{1}, M_{2} \ldots, M_{k} \subset \mathbb{R}^{n}$ be $C^{2}$ compact manifolds with or without boundary, and with $1 \leq \operatorname{dim}\left(M_{j}\right) \leq n-1$. for $j=1, \ldots, k$. Assume $0 \in \cap_{j=1}^{k} M_{j}$ and that there are $C^{2}$ smooth curves parameterized by arclength $\gamma_{i}:[0, \delta] \rightarrow M=\bigcup_{j=1}^{k} M_{j}$ with $\gamma_{i}(0)=0$ for $i=1,2, \ldots, n$ such that the one-sided derivative $\gamma_{1,+}^{\prime}(0), \ldots, \gamma_{n,+}^{\prime}(0)$ are linearly independent, hence 
$\operatorname{span}\left[\gamma_{1,+}^{\prime}(0), \gamma_{2,+}^{\prime}(0), \ldots, \gamma_{n,+}^{\prime}(0)\right]=\mathbb{R}^{n}$. Then for sufficiently large $\lambda>0$, we have that

$$
I_{\lambda}\left(0 ; M=\bigcup_{j=1}^{k} M_{j}\right) \geq \mu_{L},
$$

hence, the point 0 is a strongly extractable singular point of $M$. The constant $\mu_{L}>0$ that enters (3.10) is independent of $\lambda>0$ and is such that $C_{\lambda}^{l}\left(D_{\lambda}^{2}(\cdot ; L)\right)=1 / 2+\mu_{L}$, where $L=\bigcup_{i=1}^{n} L_{i}^{+}$with $L_{i}^{+}$the positive ray generated by the one-sided tangent vector $e_{i}:=\gamma_{i,+}^{\prime}(0)$, that is, $L_{i}^{+}=\left\{t \gamma_{i,+}^{\prime}(0), t \geq 0\right\}$, for $i=1, \ldots, n$.

Corollary 3.11. Suppose $M_{1}, M_{2}, \ldots M_{k} \subset \mathbb{R}^{n}$ be compact $C^{2}$ manifolds with $1 \leq \operatorname{dim}\left(M_{i}\right) \leq n-1, i=1,2, \ldots, k$ and assume that $0 \in \cap_{j=1}^{k} M_{j}$ with 0 a relative interior point of all $M_{j}, j=1,2, \ldots, k$. Furthermore, we assume that $M_{1}, \ldots, M_{k}$ intersect at 0 transversally, that is, $\operatorname{span}\left[T_{0} M_{1}, T_{0} M_{2}, \ldots, T_{0} M_{k}\right]=\mathbb{R}^{n}$. Then for $\lambda>0$ sufficiently large, $I_{\lambda}\left(0 ; \bigcup_{j=1}^{k} M_{j}\right)>0$.

Corollary 3.12. Suppose $M_{1}, M_{2} \subset \mathbb{R}^{n}$ be compact $C^{2}$ manifolds, with $C^{2}$ boundaries $\partial M_{1}$ and $\partial M_{2}$, satisfying $\operatorname{dim}\left(M_{1}\right)=\operatorname{dim}\left(M_{2}\right)=n-1$. Suppose that $0 \in \partial M_{1} \cap \partial M_{2}$ and assume that $\operatorname{span}\left[T_{0} M_{1}, T_{0} M_{2}\right]=\mathbb{R}^{n}$. Then for $\lambda>0$ sufficiently large, $I_{\lambda}\left(0 ; M_{1} \cup M_{2}\right)>0$.

\section{Remark 3.13.}

(a) According to the previous findings, the filter $I_{\lambda}(x ; K)$ can therefore be successfully applied to extract the transversal intersection points, for instance, in the case of:

(i) Two $C^{2}$ surfaces $M_{1}, M_{2}$ intersecting each other at a point transversally;

(ii) A surface and a curve intersecting each other transversally;

(iii) Three curves intersecting each other at a point transversally.

(b) For a piecewise affine surface $K$, such as in the case where $K$ is the boundary of a polytope in $\mathbb{R}^{3}$, by using the filter $I_{\lambda}(x ; K)$ we can also extract all the one-dimensional edges. Suppose indeed, $P_{1}$ and $P_{2}$ are two faces intersecting over an edge $L$ and assume that $p \in L$; we can then find two linearly independent vectors $e_{1}^{(i)}, e_{2}^{(i)}$ such that $p+s e_{1}^{(i)}, p+s e_{2}^{(i)} \in P_{i}$ for $i=1,2$ and $\operatorname{span}\left[e_{1}^{(1)}, e_{2}^{(1)}, e_{1}^{(2)}, e_{2}^{(2)}\right]=\mathbb{R}^{3}$. The result follows from Theorem 3.8 and the locality property Theorem 2.6. Furthermore, we have that

$$
\liminf _{\lambda \rightarrow+\infty} I_{\lambda}(x ; K)>0
$$

if and only if $x \in K$ is an edge point.

(c) We do not known whether our operator $I_{\lambda}(\cdot ; K)$ can detect tangential intersections, at least in a weak sense. One such example in $\mathbb{R}^{2}$ is the union of the two unit circles $B((0,1) ; 1)$ and $B((0,-1) ; 1)$, centred at $(0,1)$ and 
$(0,-1)$, respectively. It is then easy to check that the direction $e=(0,1)$ is a regular direction of the intersecting point $(0,0)$, hence

$$
I_{\lambda}((0,0) ; B((0,1) ; 1) \bigcup B((0,-1) ; 1))=0 .
$$

It would, however, be interesting to establish, for this case, whether near the point $(0,0)$, say at $(0, t)$ for $t>0$ small, the transform $I_{\lambda}((0, t) ; B((0,1) ; 1) \bigcup B((0,-1) ; 1))>0$. Numerical experiments appear to suggest that this is the case.

(d) Another obvious type of degeneracy is when the manifolds intersect in a non-transversal way. For example, consider two lines $l_{1}=\{(x, 0,0), x \in \mathbb{R}\}$ and $l_{2}=\{(0, y, 0), y \in \mathbb{R}\}$ in $\mathbb{R}^{3}$ that intersect at the origin. As $e_{3}=(0,0,1)$ is a regular direction of the origin, $I_{\lambda}\left((0,0,0) ; l_{1} \cup l_{2}\right)=0$.

Denote by $K=\cup_{i=1}^{m} M_{i} \subset \mathbb{R}^{n}$ the union of compact smooth manifolds and by $\mu$ a constant with $0<\mu<1$ to be taken as 'strength' of the intersection in a meaning to be made clear next. By Theorem 3.10, we may assume that there is some $\Lambda>0$ such that, when $\lambda \geq \Lambda$, the set $V_{\alpha}$ of the intersection points such that $I_{\lambda}(x ; K)=\mu_{x} \geq \mu$ for $x \in V_{\alpha}$, is not empty. By Theorem 3.4, we also know that for $\lambda$-regular points $y, I_{\lambda}(y ; K)=0$. Fixing $\lambda \geq \Lambda$ and take $K_{s} \subset \mathbb{R}^{n}$ a compact set (sample set with noise) such that

$$
\operatorname{dist}_{\mathcal{H}}\left(K, K_{s}\right) \leq \frac{\mu}{48 \sqrt{\lambda}}
$$

then by Theorem 3.1, we have that $I_{\lambda}\left(x ; K_{s}\right) \geq 3 \mu / 4$ and $I_{\lambda}\left(y ; K_{s}\right) \leq \mu / 4$. This result shows that as long as a sample set $K_{s}$ is close to $K$ we can extract 'strong intersection points' with the given strength $\mu>0$, and suppress sample noise.

We conclude this section with some observations on the singular set of a manifold by considering the general case that $K=\cup_{i=1}^{m} M_{i} \subset \mathbb{R}^{n}$ is the union of smooth compact manifolds. If we denote by $G_{\lambda}(K):=\overline{\left\{x \in \mathbb{R}^{n}, I_{\lambda}(x ; K)>0\right\}}$ the support of $I_{\lambda}(\cdot ; K)$, we may then define the essential singular set of $K$ by

$$
G_{\infty}(K)=\bigcap_{t>0} \bigcup_{\lambda \geq t} G_{\lambda}(K) .
$$

The following observations summarise what we know and what we do not know about the essential singular set $G_{\infty}(K)$.

(i) From the property of the upper transform, we infer that $G_{\lambda}(K)$ is contained in the closed $1 / \sqrt{\lambda}$ neighbourhood $K^{1 / \sqrt{\lambda}}$ of $K$. Thus $G_{\infty}(K) \subset K$.

(ii) If $x \in K$ is not an intersection point, then by Theorem 3.4, we have, for $\lambda>0$ sufficiently large, $I_{\lambda}(x ; K)=0$. Thus $x \notin G_{\infty}(K)$.

(iii) If $x \in K$ is a transversal intersection point, then Theorem 3.10 implies that for $\lambda>0$ sufficiently large, $I_{\lambda}(x ; K) \geq \mu_{x}>0$ for a constant $\mu_{x}$ independent of $\lambda$. Therefore $x \in G_{\infty}(K)$. 
(iv) If $x \in K$ is a tangential intersection of two (n-1)-dimensional smooth manifolds, we do not know whether $x \in G_{\infty}(K)$. Our numerical experiment of two tangentially intersecting spheres displayed in Figure 8(a) below suggests however that this might be the case.

$(v)$ If $x \in K$ is a non-transveral intersection point, we do not know whether $x \in G_{\infty}(K)$.

\section{Examples}

In order to verify the previous results and to gain insight into the filter $I_{\lambda}(\cdot, K)$, in this section we will examine the behaviour of $I_{\lambda}(\cdot ; K)$ for: $(i)$ two prototype examples, for which we can obtain an analytical exptression for $I_{\lambda}(\cdot ; K)$; and $(i i)$ some general geometries of intersections based on a numerical evaluation of $I_{\lambda}(\cdot ; K)$.

\subsection{Prototype models}

The two examples illustrated in this section can be considered as prototypes for intersections and sharp turning points (or edges of piecewise smooth manifolds). The first one regards the intersection between two lines, whereas the second one refers to the intersection of two rays originating at the same point.

\section{Intersection of two lines}

For a fixed $\alpha>0$, we consider the following set $K_{\alpha}=\left\{(x, y) \in \mathbb{R}^{2},|y|=\alpha|x|\right\}$.

Then for $\lambda>0$, we have for $(x, y) \in \mathbb{R}^{2}$

$$
\begin{aligned}
& C_{\lambda}^{u}\left(\chi_{K_{\alpha}}\right)(x, y)=
\end{aligned}
$$

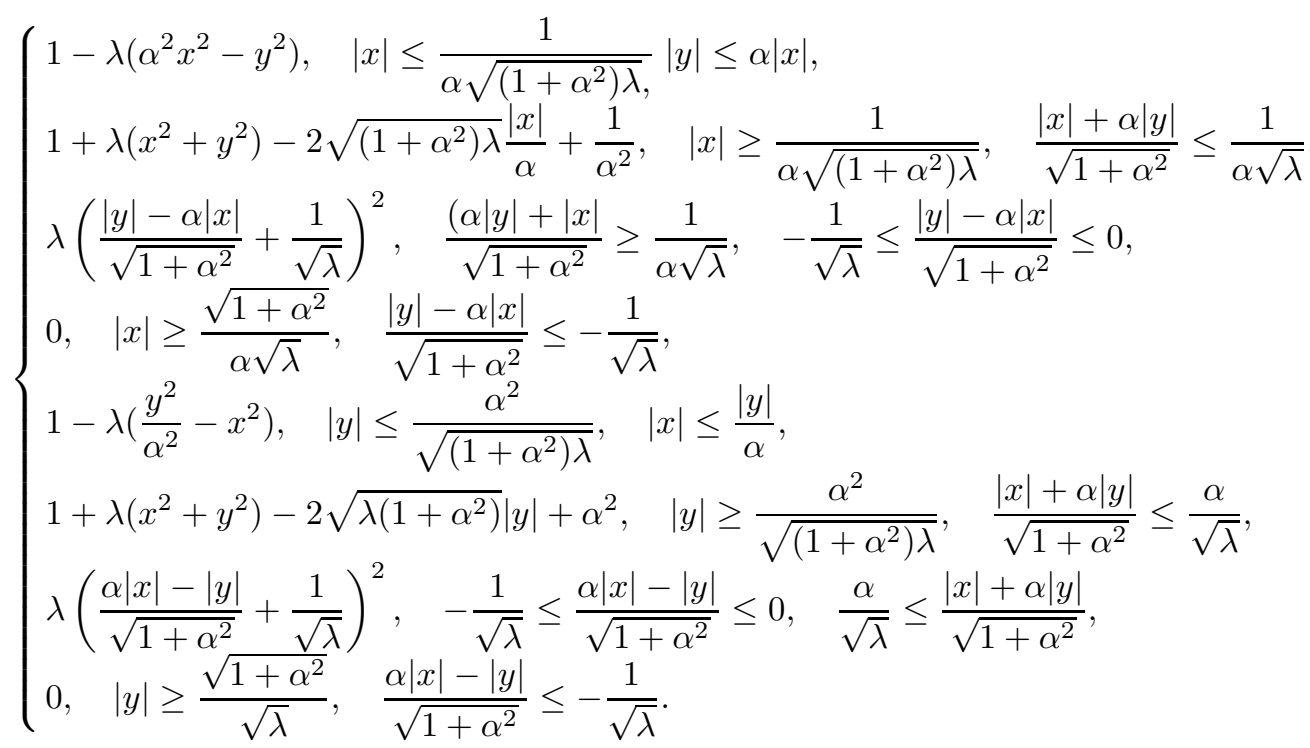


Without loss of generality, we may assume that $\alpha \geq 1$. The minimum value of $C_{\lambda}^{u}\left(\chi_{K_{\alpha}}\right)(x, y)+\lambda\left(x^{2}+y^{2}\right)$ is $1 / 2+1 /\left(2 \alpha^{2}\right)$ which is attained at $x_{ \pm}=$ $\pm \sqrt{1+\alpha^{2}} /(2 \alpha \sqrt{\lambda}), y_{ \pm}=0$. Therefore

$$
C_{\lambda}^{l}\left(C_{\lambda}^{u}\left(\chi_{K_{\alpha}}\right)\right)(0,0)=\frac{1}{2}+\frac{1}{2 \alpha^{2}}
$$

so that

$$
I_{\lambda}\left((0,0) ; K_{\alpha}\right)=\frac{1}{\alpha^{2}}
$$

We see from this example that the largest value for $I_{\lambda}\left((0,0) ; K_{\alpha}\right)$ occurs when $\alpha=1$, that is, when the two lines are perpendicular to each other. Figure 1 displays the graphs of $C_{\lambda}^{u}\left(\chi_{K_{\alpha=1}}\right)(x), C_{\lambda}^{l}\left(C_{\lambda}^{u}\left(\chi_{K_{\alpha=1}}\right)\right)(x)$ and of the filter $I_{\lambda}\left(\cdot ; K_{\alpha=1}\right)$ for this case.

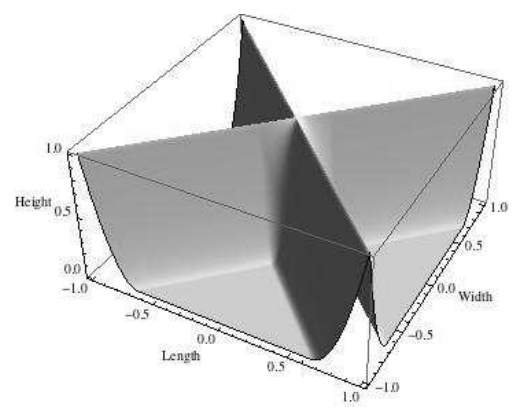

(a)

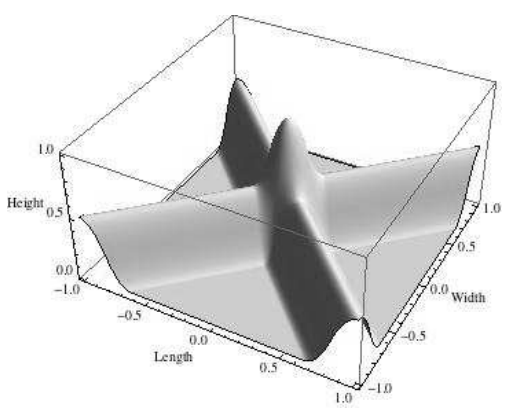

(b)

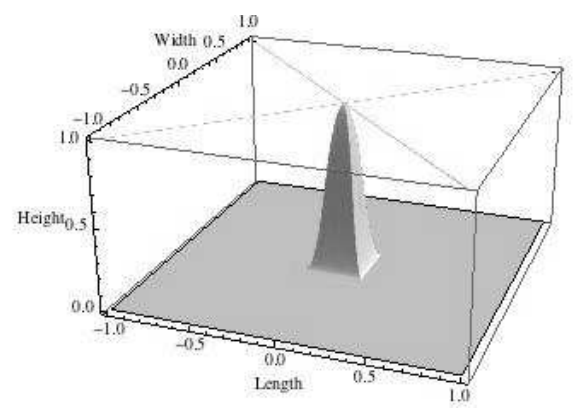

$(c)$

Fig. 1. Graph of: (a) The upper transform $C_{\lambda}^{u}\left(\chi_{K_{\alpha=1}}\right)(x)$ of the characteristic function of two crossing lines with right angle; (b) The mixed transform $C_{\lambda}^{l}\left(C_{\lambda}^{u}\left(\chi_{K_{\alpha=1}}\right)\right)(x)$; $(c)$ The intersection filter $I_{\lambda}\left(\cdot ; K_{\alpha=1}\right)$ together with the graph of the characteristic function of $K_{\alpha=1}$ displayed as reference.

Remark 4.1. Since $C_{\lambda}^{l}\left(C_{\lambda}^{u}\left(\chi_{K}\right)\right) \geq C_{\lambda}^{l}\left(D_{\lambda}^{2}(\cdot ; K)\right)$ and we have used $C_{\lambda}^{l}\left(D_{\lambda}^{2}(\cdot ; K)\right)$ to formulate sufficient conditions for the extractability of the intersection points, it is interesting to compare the 'strength' between the two filters: $I_{\lambda}\left(\cdot ; K_{\alpha}\right)$ and $J_{\lambda}\left(\cdot ; K_{\alpha}\right)$ defined by (3.3). Let us again consider the set $K_{\alpha}=\left\{(x, y) \in \mathbb{R}^{2},|y|=\right.$ $\alpha|x|\}$ and $\alpha \geq 1$. We have then

$$
\operatorname{dist}\left((x, y) ; K_{\alpha}\right)=\frac{|\alpha| x|-| y||}{\sqrt{1+\alpha^{2}}} .
$$

It is easy to show that in this case

$$
\min \left\{D_{\lambda}^{2}\left((x, y) ; K_{\alpha}\right)+\lambda\left(x^{2}+y^{2}\right),(x, y) \in \mathbb{R}^{2}\right\}=\frac{1}{2}+\frac{1}{2\left(1+2 \alpha^{2}\right)}
$$

which is attained at

$$
\left(x_{ \pm}, y_{ \pm}\right)=\left(\frac{\alpha \sqrt{1+\alpha^{2}}}{\left(1+2 \alpha^{2}\right) \sqrt{\lambda}}, 0\right)
$$


Therefore, for $\alpha \geq 1$

$$
J_{\lambda}\left((0,0) ; K_{\alpha}\right)=\frac{1}{1+2 \alpha^{2}}
$$

From the developments of the Example of the two intersecting lines, we find also that

$$
I_{\lambda}\left((0,0) ; K_{\alpha}\right)=\frac{1}{\alpha^{2}}
$$

hence, $I_{\lambda}\left((0,0) ; K_{\alpha}\right)>J_{\lambda}\left((0,0) ; K_{\alpha)}\right.$. In particular, for $\alpha=1, J_{\lambda}\left((0,0) ; K_{1}\right)=1 / 3$ while $I_{\lambda}\left((0,0) ; K_{1}\right)=1$. This shows that $I_{\lambda}(\cdot ; K)$ is a much stronger filter than $J_{\lambda}(\cdot ; K)$.

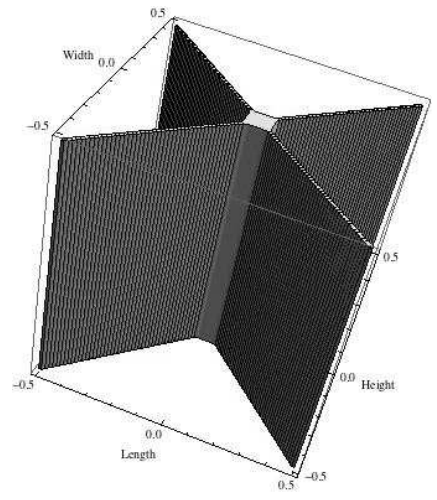

(a)

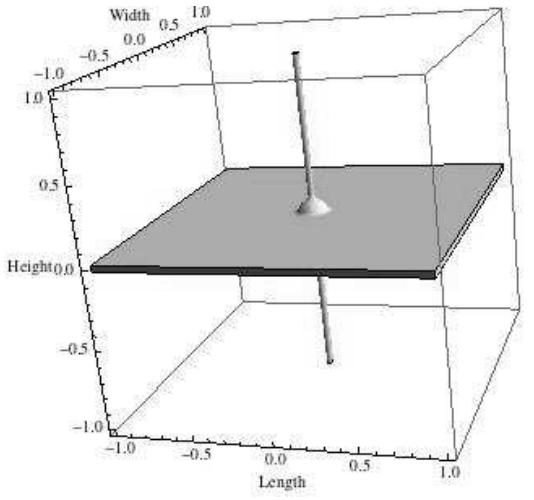

(b)

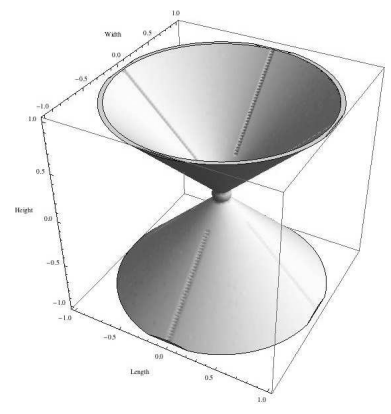

(c)

Fig. 2. (a) Marker $I_{\lambda}\left(\cdot ; P_{1} \cup P_{2}\right)$ of two intersecting planes $P_{1}$ and $P_{2}$ at right angles with threshold $0.75, \lambda=25$ and the characteristic function of the set as reference; $(b)$ Marker $I_{\lambda}(\cdot ; P \cup L)$ of plane $P$ intersecting line $L$ at right angles with threshold 0.75 and $\lambda=25$. (c) Marker of the singularity of a cone with $\lambda=25$;

Remark 4.2. The example of two intersecting lines can be used to construct prototypes in $\mathbb{R}^{3}$, such as the intersection of two planes, by applying Proposition 2.11, and the cone, by applying Proposition 2.10. The graph of the filter $I_{\lambda}(\cdot ; K)$ for these two cases is displayed in Figure 2. 


\section{Intersection of two rays}

For a fixed $\alpha>0$, let us consider now the set $K_{\alpha}^{+}=\left\{(x, y) \in \mathbb{R}^{2}, x \geq 0,|y| \leq \alpha x\right\}$. For $\lambda>0$, we have for $(x, y) \in \mathbb{R}^{2}$

$$
\begin{aligned}
& C_{\lambda}^{u}\left(\chi_{K_{\alpha}^{+}}\right)(x, y)= \\
& \left\{\begin{array}{l}
1-\left(\lambda \alpha^{2} x^{2}-\lambda y^{2}\right), \quad|y| \leq \alpha x, 0 \leq x \leq \frac{1}{\alpha \sqrt{\left(1+\alpha^{2}\right) \lambda}}, \\
0, \quad \frac{|y|-\alpha x}{\sqrt{1+\alpha^{2}}} \leq-\frac{1}{\sqrt{\lambda}}, \quad x \geq \frac{\sqrt{1+\alpha^{2}}}{\alpha \sqrt{\lambda}}, \\
\lambda\left(\frac{1}{\sqrt{\lambda}}+|y|-\alpha x \sqrt{1+\alpha^{2}}\right)^{2}, \quad-\frac{1}{\sqrt{\lambda}} \leq \frac{|y|-\alpha x}{\sqrt{1+\alpha^{2}}} \leq 0, \frac{1}{\alpha \sqrt{\lambda}} \leq \frac{\alpha|y|+x}{\sqrt{1+\alpha^{2}}}, \\
-\left(\frac{2 x \sqrt{\left(1+\alpha^{2}\right) \lambda}}{\alpha}-\frac{1}{\alpha^{2}}\right)+\lambda\left(x^{2}+y^{2}\right)+1, \quad x \geq \frac{1}{\alpha \sqrt{\left(1+\alpha^{2}\right) \lambda}}, \frac{\alpha|y|+x}{\sqrt{1+\alpha^{2}}} \leq \frac{1}{\alpha \sqrt{\lambda}}, \\
\lambda\left(-\frac{1}{\sqrt{\lambda}}+\frac{|y|-\alpha x}{\sqrt{1+\alpha^{2}}}\right)^{2}, \quad 0 \leq \frac{|y|-\alpha x}{\sqrt{1+\alpha^{2}}} \leq \frac{1}{\sqrt{\lambda}}, \frac{\alpha|y|+x}{\sqrt{1+\alpha^{2}}} \geq 0, \\
\lambda\left(\sqrt{x^{2}+y^{2}}-\frac{1}{\sqrt{\lambda}}\right)^{2}, \quad \sqrt{x^{2}+y^{2}} \leq \frac{1}{\sqrt{\lambda}}, \frac{(\alpha|y|+x)}{\sqrt{1+\alpha^{2}}} \leq 0, \\
0, \quad \frac{(\alpha|y|+x)}{\sqrt{1+\alpha^{2}}} \geq 0, \frac{|y|-\alpha x}{\sqrt{1+\alpha^{2}}} \geq \frac{1}{\sqrt{\lambda}}, \\
0, \quad \sqrt{x^{2}+y^{2}} \geq \frac{1}{\sqrt{\lambda}}, \quad \frac{(\alpha|y|+x)}{\sqrt{1+\alpha^{2}}} \leq 0 .
\end{array}\right.
\end{aligned}
$$

The formula for the mixed transform $C_{\lambda}^{l}\left(C_{\lambda}^{u}\left(\chi_{K_{\alpha}^{+}}\right)\right)(x, y)$ is very complicated and is omitted here. There is a critical angle $\alpha_{c}=\sqrt{(\sqrt{5}-1) / 2}$ which is the positive solution of $\alpha^{4}+\alpha^{2}=1$. We have the explicit formulae for the case $\alpha \geq \alpha_{c}$, whereas for $\alpha \leq \alpha_{c}$ we have not yet derived the complete expressions of $C_{\lambda}^{l}\left(C_{\lambda}^{u}\left(\chi_{K_{\alpha}^{+}}\right)\right)(x, y)$. However, at the intersection (sharp turning point) $(0,0)$, we have,

$$
C_{\lambda}^{l}\left(C_{\lambda}^{u}\left(\chi_{K_{\alpha}^{+}}\right)\right)(0,0)= \begin{cases}\frac{1+\alpha^{2}}{2+\alpha^{2}}, & \alpha \leq \alpha_{c}, \\ \frac{1+\alpha^{2}}{\alpha^{2}}-\frac{2\left(1+\alpha^{2}\right)\left(\sqrt{1+\alpha^{2}}-\alpha\right)^{2}}{\alpha^{2}}, & \alpha \geq \alpha_{c} .\end{cases}
$$

In both cases we have $C_{\lambda}^{l}\left(C_{\lambda}^{u}\left(\chi_{K_{\alpha}^{+}}\right)\right)(0,0)>1 / 2$. We also see that when $\alpha \rightarrow 0_{+}$

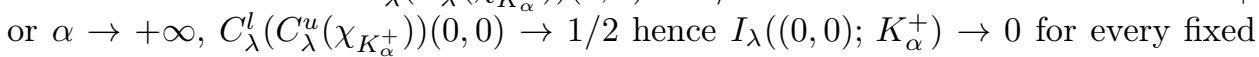
$\lambda>0$. The behaviour of $I_{\lambda}\left(\cdot ; K_{\alpha}^{+}\right)$can be appreciated by inspecting Figure 3 and Figure 4. Figure 3(a) displays the scaled graph of $I_{\lambda}\left((0,0) ; K_{\alpha}^{+}\right)$for $0 \leq \alpha \leq 3$ with its maximum value equal approximately to 0.260396 attained at $\alpha=0.9227$, whereas Figure $3(b)$ shows the graph of $I_{\lambda}\left((x, 0) ; K_{\alpha}^{+}\right)$for $\alpha=1$ and $\lambda=25$. Figure 4 displays the graphs, for the case $\alpha=1>\alpha_{c}$ and $\lambda=25$, of $C_{\lambda}^{u}\left(\chi_{K_{\alpha}^{+}}\right)$, $C_{\lambda}^{l}\left(C_{\lambda}^{u}\left(\chi_{K_{\alpha}^{+}}\right)\right), I_{\lambda}\left(\cdot ; K_{\alpha}^{+}\right)$and of the scaled $I_{\lambda}\left(\cdot ; K_{\alpha}^{+}\right)$. We also observe that the maximum of $I_{\lambda}\left(\cdot ; K_{\alpha}^{+}\right)$is not attained at the point $(0,0)$.

\section{Remark 4.3.}




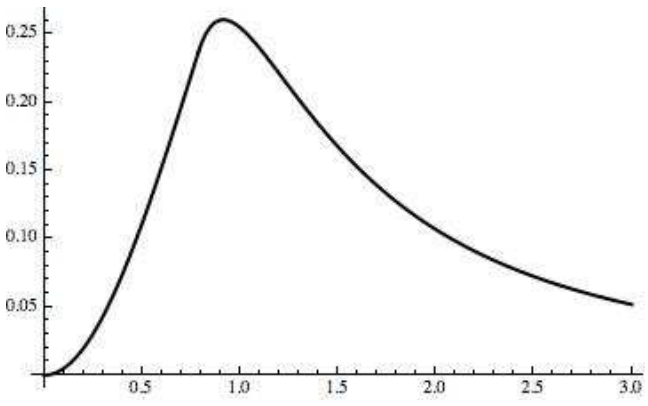

(a)

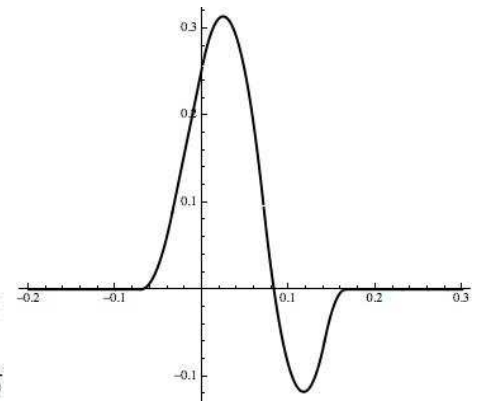

(b)

Fig. 3. (a) The graph of $\alpha \mapsto I_{\lambda}\left((0,0) ; K_{\alpha}^{+}\right)$; (b): The graph of $x \mapsto I_{\lambda}\left((x, 0) ; K_{\alpha}^{+}\right)$for $\alpha=1$ and $\lambda=25$.

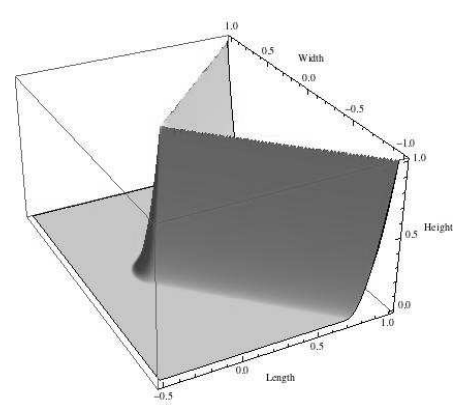

(a)

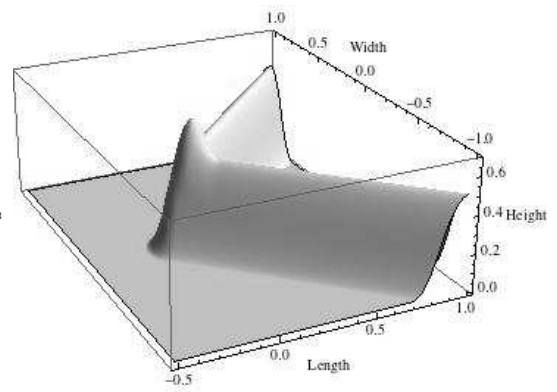

(b)

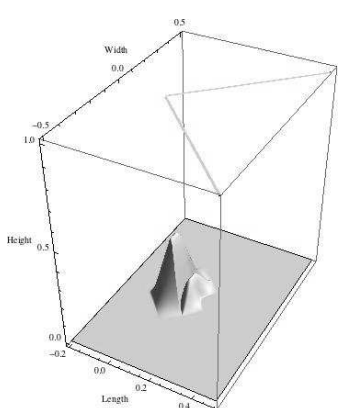

(c)

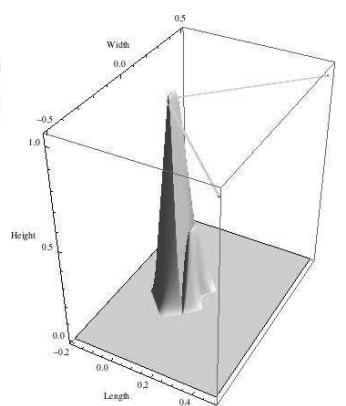

$(d)$

Fig. 4. (a) The upper transform $C_{\lambda}^{u}\left(\chi_{K_{\alpha}^{+}}\right)$of the characteristic function of two crossing rays with right angle $(\alpha=1)$ and $\lambda=25$; (b) The mixed transform $C_{\lambda}^{l}\left(C_{\lambda}^{u}\left(\chi_{K_{\alpha}^{+}}\right)\right)$; (c) The intersection marker given by $I_{\lambda}\left(\cdot ; K_{\alpha}^{+}\right)$and the characteristic function; $(d)$ Scaled intersection marker with the characteristic function $\chi_{K_{\alpha}^{+}}$as reference.

(a) From the graph of the mapping $\alpha \mapsto I_{\lambda}\left((0,0) ; K_{\alpha}^{+}\right)$, we see that if $\alpha>0$ is small or large, $I_{\lambda}\left((0,0) ; K_{\alpha}^{+}\right)$will be small, hence with this meaning, we can say that the filter $I_{\lambda}\left(\cdot ; K_{\alpha}^{+}\right)$captures somehow the strength of the different intersections and sharp turning points.

(b) Figure 4 shows that the intersection marker $I_{\lambda}\left(\cdot ; K_{\alpha}^{+}\right)$lies on the interior corner formed by the two lines. The numerical experiments described in Section 4.2 agree with this prototype for turning points.

Remark 4.4. Even this example can be used to construct prototype for intersections in $\mathbb{R}^{3}$, such as the intersection of two semiplanes by applying Proposition 2.11, and the vertex of a convex cone, by applying Proposition 2.10. Suplevel set of the scaled filter $I_{\lambda}(x ; K)$ (i.e. after normalizing it to one) are displayed in Figure 5 alon 


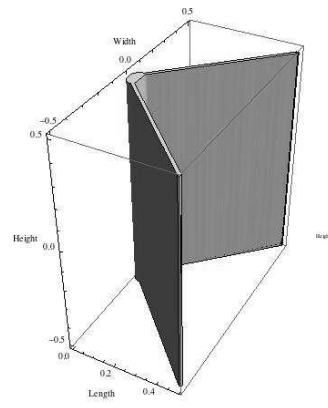

(a)

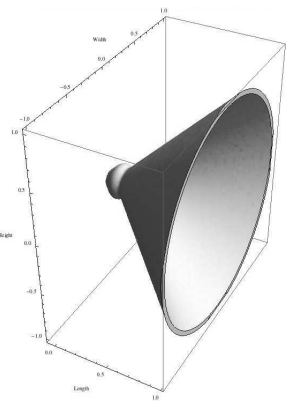

(b)

Fig. 5. (a) Marker of singularities for a surface formed by two planes with right angle and $\lambda=25$; (b) Marker of singularity of a convex cone with $\lambda=25$ and with the characteristic function as reference.

with the suplevel set of the characteristic function of the object.

\subsection{Numerical Experiments}

For more complicated geometries, the filter $I_{\lambda}(\cdot ; K)$ must be evaluated numerically. The numerical realization of $I_{\lambda}(\cdot ; K)$ relies on the availability of numerical schemes for computing the upper and lower transform of a given function, which then means the availability of schemes to compute the convex envelope of a function. We refer to Ref. 28 for the algorithmic and implementation details of the scheme we have adopted, by just noting here that, because of the locality property of the compensated convex transforms (see for instance Theorem 2.6 where quantitative estimates of the neighborhood size are also given), it is possible to develop fast schemes that depend only on the local behaviour of the input function. This is in sharp contrast to the evaluation of the convex envelope of a function which is a global evaluation.

In this section, we will illustrate some numerical experiments which are only meant to show the effectiveness of the filter $I_{\lambda}(\cdot ; K)$ and its Hausdorff stability property. We will consider both $2 d-$ and $3 d$-geometries. The geometry is digitized and input as an image, but also other computer representations of the geometry can clearly be handled. This depends finally on the representation of the input geometry for the numerical scheme that is used to compute the compensated transforms. Figure 6 is an instance of a set of $2 d$ curves which intersect to each other in different manner. The Figure shows the position of the local maxima of $I_{\lambda}(\cdot ; K)$ which are seen to coincide with all the crossing and turning points of the given curves.

Figure 7 displays the results of the application of the filter $I_{\lambda}(\cdot ; K)$ to $3 d$ geometries represented by point clouds. Figure $7(a)$ displays a $3 \mathrm{~d}$ frame with different type of connections, whereas Figure 7(b) depicts the intersections between manifolds of different dimensions, namely, in the Figure a sphere, a plane and a line, intersecting each other, are considered. Figure $7(c)$ shows the Whitney umbrella of the implicit 


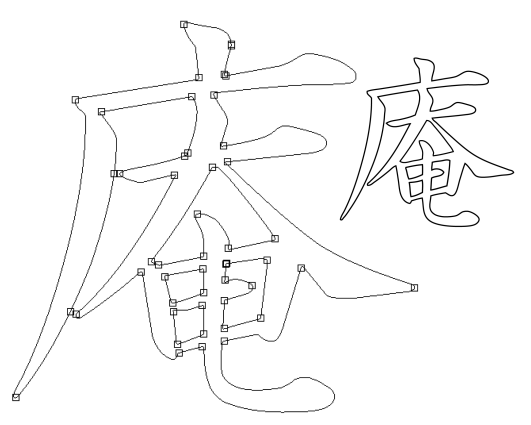

(a)

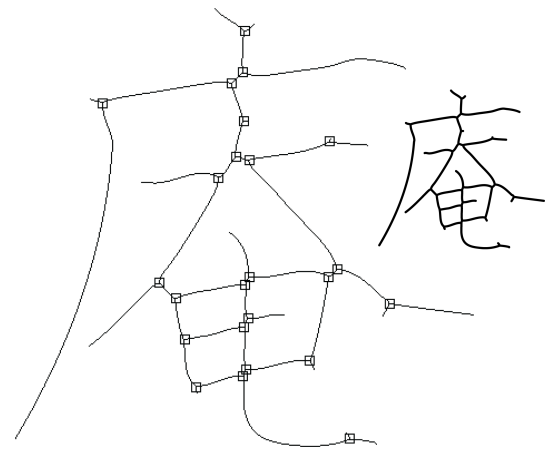

(b)

Fig. 6. (a) Sharp turning point of curves; (b) Intersection points of medial axis curves.

equation $x^{2}=y^{2} z$, with the location of where the surface intersects itself.

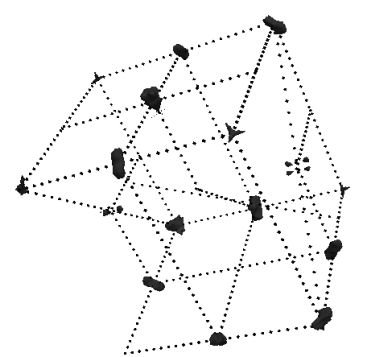

$(a)$

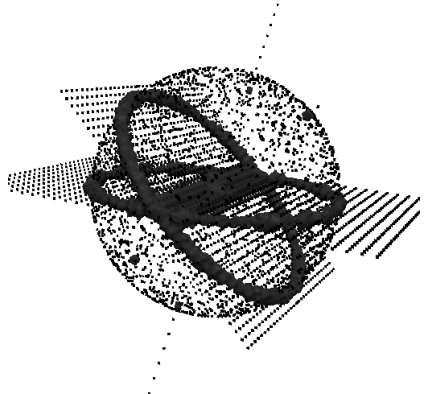

(b)

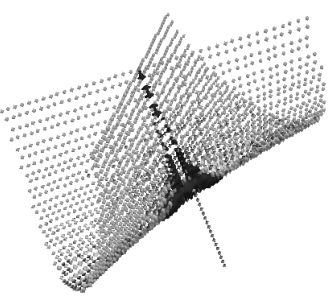

$(c)$

Fig. 7. (a) Intersections of sampled line segments in $3 d$ with different ways of connections; $(b)$ Intersections of sampled sphere, planes and lines; $(c)$ Self intersections of the sampled Whitney umbrella given by the equation $x^{2}=y^{2} z$.

The numerical experiments displayed in Figure 8 and Figure 9 refer to critical conditions that are not directly covered by the theoretical results we have obtained. Figure 8 shows the result of the application of $I_{\lambda}(\cdot ; K)$ to two spheres that are 'almost' tangentially intersecting each other, whereas Figure 9 illustrates the results of the application of the filter to detect the intersection between loosely sampled piecewise affine functions, a plane and a line. Different suplevel sets of $I_{\lambda}(\cdot ; K)$, reflecting the different strength of the type of intersection are shown in Figure 9. For instance, the intersection of the line with the plane for the geometry shown in 
Figure 9 is weaker than the geometric singularities of the piecewise affine surface. With this meaning, the values of the local maxima of $I_{\lambda}(\cdot ; K)$ determine a scale between the different type of intersections present in the manifold $K$ and represents the multiscale nature of the filter $I_{\lambda}(\cdot ; K)$.

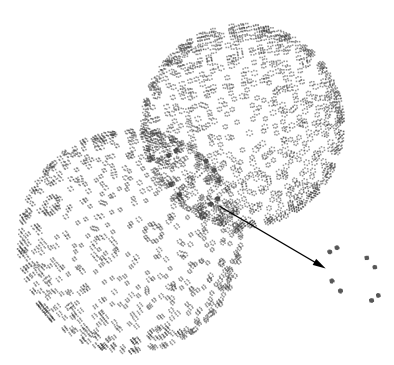

Fig. 8. Two sampled spheres, with equation $\left\{(i, j, k):\left|(i-50)^{2}+(j-50)^{2}+(k-120)^{2}-30^{2}\right|<=1\right\}$ and $\left\{(i, j, k):\left|(i-50)^{2}+(j-50)^{2}+(k-50)^{2}-40^{2}\right| \leq 1\right\}$, which are 'almost' tangentially intersected, and indication of the intersection marker.

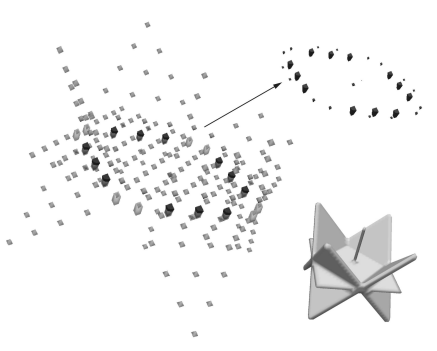

(a)

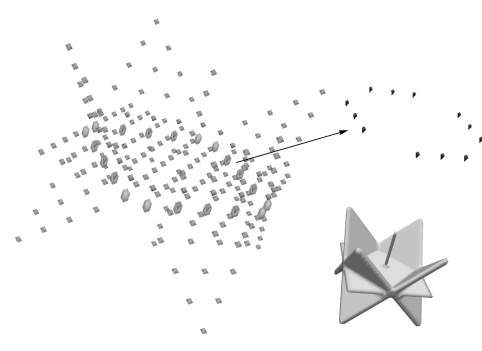

(b)

Fig. 9. Intersection markers for the intersection among loosely sampled piecewise affine surfaces of equation || $10 x-75|-| 10 y-75|+| 10 z-75|-45|=0$, the plane of equation $y=22$ and the line of equation $x=22, z=22$. The figure displays different suplevel sets of $I_{\lambda}(x ; K):(a)$ $I_{\lambda}(x ; K) \geq 0.40 \max _{x \in K}\left(I_{\lambda}(x ; K)\right) ;(b) I_{\lambda}(x ; K) \geq 0.65 \max _{x \in K}\left(I_{\lambda}(x ; K)\right)$.

\section{Proofs of the Main Results}

Proof of Proposition 2.11. Part $(i)$ Since $\chi_{K_{m}^{n}}(x, z)=\chi_{K_{m}}(x)$ for all $x \in \mathbb{R}^{m}$, $z \in \mathbb{R}^{n}$, the application of Proposition 2.9 yields

$$
\mathrm{co}_{\mathbb{R}^{n+m}}\left[\lambda|x|^{2}+\lambda|z|^{2}-\chi_{K_{m}^{n}}\right](x, z)=\lambda|z|^{2}+\operatorname{co}_{\mathbb{R}^{m}}\left[\lambda|x|^{2}-\chi_{K_{m}}\right](x)
$$


which proves $(2.12)_{1}$. The proof of $(2.12)_{2}$, is also easy to verify using the definition of $D_{\lambda}^{2}(\cdot ; K)$.

Part (ii): The proof of $(2.12)_{2}$ follows from the definition of $D_{\lambda}^{2}(\cdot ; K)$. As for the proof of $(2.12)_{1}$, by the definition of upper transform, we have

$$
\operatorname{co}\left[\lambda|x|^{2}-\chi_{K_{m}}\right](x)=\lambda|x|^{2}-C_{\lambda}^{u}\left(\chi_{K_{m}}\right)(x), \quad x \in \mathbb{R}^{m} .
$$

We need to show that this is also the convex envelope of the function $\lambda\left(|x|^{2}+|z|^{2}\right)-$ $\chi_{K_{m}^{0, n}}(x, z)$ at $z=0$. By definition,

$$
\begin{aligned}
\lambda|x|^{2}-C_{\lambda}^{u}\left(\chi_{K_{m}}\right)(x)=\operatorname{co}\left[\lambda|x|^{2}-\chi_{K_{m}}\right](x) & \leq \lambda|x|^{2}-\chi_{K_{m}}(x) \\
& \leq \lambda\left(|x|^{2}+|z|^{2}\right)-\chi_{K_{m}^{0, n}}(x, z) .
\end{aligned}
$$

Thus for $z=0$,

$$
\operatorname{co}\left[\lambda|x|^{2}-\chi_{K_{m}}\right](x) \leq \operatorname{co}\left[\lambda\left(|x|^{2}+|z|^{2}\right)-\chi_{K_{m}^{0, n}}\right](x, z=0) .
$$

On the other hand, by restricting to the plane $z=0$, we obtain

$\operatorname{co}\left[\lambda\left(|x|^{2}+|z|^{2}\right)-\chi_{K_{m}^{0, n}}\right](x, z=0) \leq \lambda\left(|x|^{2}+|z|^{2}\right)-\left.\chi_{K_{m}^{0, n}}|(x, z=0)=\lambda| x\right|^{2}-\chi_{K_{m}}(x)$.

As restrictions of convex functions in a subspace remain convex, by definition of the convex envelope, we find

$$
\operatorname{co}\left[\lambda\left(|x|^{2}+|z|^{2}\right)-\chi_{K_{m}^{0, n}}\right](x, z=0) \leq \operatorname{co}\left[\lambda|x|^{2}-\chi_{K_{m}}\right](x),
$$

which proves $(2.13)_{1}$.

Proof of Lemma 2.13 It was proved in Lemma 5.4 of Ref. 27 that

$$
\left.\left.\mid D_{\lambda}^{2}(x ; E)\right)-D_{\lambda}^{2}(x ; F)\right) \mid \leq 2 \sqrt{\lambda} \operatorname{dist}_{\mathcal{H}}(E, F),
$$

and thus

$$
D_{\lambda}^{2}(x ; F)-\operatorname{dist}_{\mathcal{H}}(E, F) \leq D_{\lambda}^{2}(x ; E) \leq D_{\lambda}^{2}(x ; F)+\operatorname{dist}_{\mathcal{H}}(E, F) .
$$

Taking the lower transform in (5.7) and using the ordering property of lower transforms, we obtain

$C_{\lambda}^{l}\left(D_{\lambda}^{2}(\cdot ; F)-\operatorname{dist}_{\mathcal{H}}(E, F)\right)(x) \leq C_{\lambda}^{l}\left(D_{\lambda}^{2}(\cdot ; E)\right)(x) \leq C_{\lambda}^{l}\left(D_{\lambda}^{2}(\cdot ; F)+\operatorname{dist}_{\mathcal{H}}(E, F)\right)(x)$.

Then by the affine covariant property $\operatorname{co}[f+\ell]=\operatorname{co}[f]+\ell$, where $\ell$ an affine function, of the convex envelope, we have that

$C_{\lambda}^{l}\left(D_{\lambda}^{2}(\cdot ; F)\right)(x)-\operatorname{dist}_{\mathcal{H}}(E, F) \leq C_{\lambda}^{l}\left(D_{\lambda}^{2}(\cdot ; E)\right)(x) \leq C_{\lambda}^{l}\left(D_{\lambda}^{2}(\cdot ; F)\right)(x)+\operatorname{dist}_{\mathcal{H}}(E, F)$, from which the result follows.

Proof of Theorem 3.1. The statement follows from the expression of $I_{\lambda}(\cdot ; K)$ in terms of the stable ridge transform (1.6) and from the Hausdorff stability of the 
upper transform and of the stable ridge transform, Theorem 2.12, after applying the triangle inequality.

Proof of Theorem 3.4. We first establish the result in $\mathbb{R}^{2}$ and then apply Proposition 2.10 to generalise the result to higher dimensional cases. Without loss of generality, we may assume that $x=(0,0)$ and $e=(0,1) \in \mathbb{R}^{2}$. By our assumption that $e$ is a $\delta$-regular direction, we have $\bar{B}((0, \delta) ; \delta) \cap K=\{(0,0)\}$ and $\bar{B}((0,-\delta) ; \delta) \cap K=\{(0,0)\}$. Now we define $K_{+}=B((0,-\delta) ; \delta) \cup B^{c}((0, \delta) ; \delta)$ and $K_{-}=\{(0,0)\}$, then $\chi_{K_{-}}(x, y) \leq \chi_{K}(x, y) \leq \chi_{K_{+}}(x, y)$ so that

$$
C_{\lambda}^{u}\left(\chi_{K_{-}}\right)(x, y) \leq C_{\lambda}^{u}\left(\chi_{K}\right)(x, y) \leq C_{\lambda}^{u}\left(\chi_{K_{+}}\right)(x, y)
$$

and

$$
C_{\lambda}^{l}\left(C_{\lambda}^{u}\left(\chi_{K_{-}}\right)\right)(x, y) \leq C_{\lambda}^{l}\left(C_{\lambda}^{u}\left(\chi_{K}\right)\right) \leq C_{\lambda}^{l}\left(C_{\lambda}^{u}\left(\chi_{K_{+}}\right)\right)(x, y) .
$$

We will show that for $t \in[-\delta, \delta]$ and for $\lambda>1 / \delta^{2}$,

$$
C_{\lambda}^{l}\left(C_{\lambda}^{u}\left(\chi_{K_{-}}\right)\right)(0, t)=C_{\lambda}^{l}\left(C_{\lambda}^{u}\left(\chi_{K_{+}}\right)\right)(0, t) .
$$

First we have

$$
C_{\lambda}^{u}\left(\chi_{K_{-}}\right)(x, y)= \begin{cases}\lambda\left(1 / \sqrt{\lambda}-\sqrt{x^{2}+y^{2}}\right)^{2}, \sqrt{x^{2}+y^{2}} \leq 1 / \sqrt{\lambda} \\ 0, & \sqrt{x^{2}+y^{2}} \geq 1 / \sqrt{\lambda}\end{cases}
$$

The formula for $C_{4 \lambda}^{u}\left(\chi_{K_{-}}\right)(x, y)$ is similar and is obtained by just replacing $\lambda$ by $4 \lambda$ in (5.11). We also have,

$$
C_{\lambda}^{l}\left(C_{\lambda}^{u}\left(\chi_{K_{-}}\right)\right)(x, y)\left\{\begin{array}{l}
\frac{1}{2}-\lambda\left(x^{2}+y^{2}\right), \sqrt{x^{2}+y^{2}} \leq \frac{1}{2 \sqrt{\lambda}} \\
C_{\lambda}^{u}\left(\chi_{K_{-}}\right)(x, y), \sqrt{x^{2}+y^{2}} \geq \frac{1}{2 \sqrt{\lambda}}
\end{array}\right.
$$

For $K_{+}$and for $\lambda \geq 1 / \delta^{2}$, we have, by the regular extension theorem (see Theorem 3.4 of Ref. 27), that

$C_{\lambda}^{u}\left(\chi_{K_{+}}\right)(x, y)= \begin{cases}1, & (x, y) \in K_{+}, \\ \lambda\left(\delta-1 / \sqrt{\lambda}-\sqrt{x^{2}+(|y|-\delta)^{2}}\right)^{2}, & \delta-1 / \sqrt{\lambda} \leq \sqrt{x^{2}+(|y|-\delta)^{2}} \leq \delta, \\ 0, & \sqrt{x^{2}+(|y|-\delta)^{2}} \leq \delta-1 / \sqrt{\lambda} .\end{cases}$

Thus for $|y| \leq \delta$, we have

$$
C_{\lambda}^{u}\left(\chi_{K_{-}}\right)(0, y)=C_{\lambda}^{u}\left(\chi_{K}\right)(0, y)=C_{\lambda}^{u}\left(\chi_{K_{+}}\right)(0, y)
$$

and

$$
C_{4 \lambda}^{u}\left(\chi_{K_{-}}\right)(0, y)=C_{4 \lambda}^{u}\left(\chi_{K}\right)(0, y)=C_{4 \lambda}^{u}\left(\chi_{K_{+}}\right)(0, y)
$$


Now we calculate $C_{\lambda}^{l}\left(C_{\lambda}^{u}\left(\chi_{K_{+}}\right)\right)(0, y)$ for $y \in[-\delta, \delta]$. First we consider the function of a single variable

$$
g(y):=C_{\lambda}^{u}\left(\chi_{K_{+}}\right)(0, y)= \begin{cases}\lambda(1 / \sqrt{\lambda}-|y|)^{2}, & |y| \leq 1 / \sqrt{\lambda}, \\ 0, & 1 / \sqrt{\lambda} \leq|y| \leq 2 \delta-1 / \sqrt{\lambda}, \\ \lambda(2 \delta-1 / \sqrt{\lambda}-|y|)^{2}, & 2 \delta-1 / \sqrt{\lambda} \leq|y| \leq 2 \delta, \\ 1, & |y| \geq 2 \delta .\end{cases}
$$

Now we consider the function

$$
f_{\lambda}(y)= \begin{cases}\frac{1}{2}, & |y| \leq \frac{1}{2 \sqrt{\lambda}}, \\ \lambda(1 / \sqrt{\lambda}-|y|)^{2}+\lambda y^{2}, & \frac{1}{2 \sqrt{\lambda}} \leq|y| \leq \frac{1}{\sqrt{\lambda}}, \\ \lambda y^{2}, & |y| \geq \frac{1}{\sqrt{\lambda}} .\end{cases}
$$

It is easy to verify that $f_{\lambda}(y)$ is convex and $f_{\lambda}(y)=\operatorname{co}\left[g+\lambda|y|^{2}\right](y)$ for $y \in[-\delta, \delta]$. Now we show that $f_{\lambda}(y) \leq C_{\lambda}^{u}\left(\chi_{K_{+}}\right)(x, y)+\lambda\left(x^{2}+y^{2}\right)$ for $(x, y) \in \mathbb{R}^{2}$. To this purpose, we consider first the case when $\sqrt{x^{2}+(|y|-\delta)^{2}} \geq \delta$; i.e. $x \in K_{+}$. Then we have

$$
C_{\lambda}^{u}\left(\chi_{K_{+}}\right)(x, y)+\lambda\left(x^{2}+y^{2}\right)=1+\lambda\left(x^{2}+y^{2}\right) \geq 1+\lambda y^{2} .
$$

Now, for $|y| \leq 1 /(2 \sqrt{\lambda})$, we have that

$$
1+\lambda y^{2} \geq 1 / 2=f_{\lambda}(y),
$$

for $1 /(2 \sqrt{\lambda}) \leq|y| \leq 1 / \sqrt{\lambda}$, we get that

$$
f_{\lambda}(y) \leq f_{\lambda}(1 / \sqrt{\lambda})=1 \leq 1+\lambda y^{2},
$$

whereas for $|y| \geq 1 / \sqrt{\lambda}$, we find

$$
f_{\lambda}(y)=\lambda y^{2} \leq 1+\lambda y^{2} .
$$

By comparing these results, we conclude therefore that when $\sqrt{x^{2}+(|y|-\delta)^{2}} \geq \delta$,

$$
f_{\lambda}(y) \leq C_{\lambda}^{u}\left(\chi_{K_{+}}\right)(x, y)+\lambda\left(x^{2}+y^{2}\right) .
$$

On the other hand, when $\sqrt{x^{2}+(|y|-\delta)^{2}} \leq \delta-1 / \sqrt{\lambda}$, we can easily verify that

$$
1 / \sqrt{\lambda} \leq|y| \leq 2 \delta-1 / \sqrt{\lambda}
$$

so that

$$
f_{\lambda}(y)=\lambda y^{2} \leq \lambda\left(x^{2}+y^{2}\right)=C_{\lambda}^{u}\left(\chi_{K_{+}}\right)(x, y)+\lambda\left(x^{2}+y^{2}\right) .
$$

What remains is to see what happens when $\delta-1 / \sqrt{\lambda}<\sqrt{x^{2}+(|y|-\delta)^{2}}<\delta$. To this purpose, we define $U_{\lambda}=\left\{(x, y) \in \mathbb{R}^{2}, \delta-1 / \sqrt{\lambda}<\sqrt{x^{2}+(|y|-\delta)^{2}}<\delta\right\}$. If $(x, y) \in U_{\lambda}$ and $x \neq 0$, it is easy to see that

$$
\operatorname{sign}\left(\frac{\partial}{\partial x}\left(C_{\lambda}^{u}\left(\chi_{K_{+}}\right)(x, y)+\lambda\left(x^{2}+y^{2}\right)\right)\right)=\operatorname{sign}(x)
$$


where $\operatorname{sign}(\cdot)$ is the signature function. Thus $C_{\lambda}^{u}\left(\chi_{K_{+}}\right)(x, y)+\lambda\left(x^{2}+y^{2}\right)$ is increasing for $x>0$ and decreasing for $x<0$ in the region $U_{\lambda}$. Thus if $|y| \leq 1 / \sqrt{\lambda}$ or $2 \delta-1 / \sqrt{\lambda} \leq|y| \leq 2 \delta$ and $(x, y) \in U_{\lambda}$,

$$
C_{\lambda}^{u}\left(\chi_{K_{+}}\right)(x, y)+\lambda\left(x^{2}+y^{2}\right) \geq g(y)+\lambda y^{2} \geq f_{\lambda}(y) .
$$

If $1 / \sqrt{\lambda} \leq|y| \leq 2 \delta-1 / \sqrt{\lambda}$ and $(x, y) \in U_{\lambda}$, then

$$
C_{\lambda}^{u}\left(\chi_{K_{+}}\right)(x, y)+\lambda\left(x^{2}+y^{2}\right) \geq \lambda y^{2}=f_{\lambda}(y) .
$$

In summary, for all $(x, y) \in \mathbb{R}^{2}$, the convex function $f_{\lambda}(y)$ satisfies

$$
\begin{aligned}
& f_{\lambda}(y) \leq C_{\lambda}^{u}\left(\chi_{K_{+}}\right)(x, y)+\lambda\left(x^{2}+y^{2}\right) \\
& \text { and } \quad f_{\lambda}(y)=C_{\lambda}^{u}\left(\chi_{K_{+}}\right)(0, y)+\lambda y^{2}
\end{aligned}
$$

for $1 /(2 \sqrt{\lambda}) \leq|y| \leq \delta$, whereas $f_{\lambda}(y)=1 / 2$ when $|y| \leq 1 /(2 \sqrt{\lambda})$, and

$$
f( \pm 1 /(2 \sqrt{\lambda}))=C_{\lambda}^{u}\left(\chi_{K_{+}}\right)\left(0, \pm 1 /(2 \sqrt{\lambda})+\lambda(1 /(2 \sqrt{\lambda}))^{2}=1 / 2 .\right.
$$

Therefore

$$
f_{\lambda}(y)=\operatorname{co}\left[C_{\lambda}^{u}\left(\chi_{K_{+}}\right)+\lambda\left(x^{2}+y^{2}\right)\right](x=0, y)
$$

for $|y| \leq \delta$. Thus

$$
f_{\lambda}(y)-\lambda y^{2}=C_{\lambda}^{l}\left(C_{\lambda}^{u}\left(\chi_{K_{+}}\right)\right)(x=0, y) .
$$

However, by a direct comparison we also see that

$$
f_{\lambda}(y)-\lambda y^{2}=C_{\lambda}^{l}\left(C_{\lambda}^{u}\left(\chi_{K_{-}}\right)\right)(x=0, y) .
$$

Consequently

$$
C_{\lambda}^{l}\left(C_{\lambda}^{u}\left(\chi_{K}\right)\right)(0, y)=f_{\lambda}(y)-\lambda y^{2}= \begin{cases}\frac{1}{2}-\lambda y^{2}, & |y| \leq \frac{1}{2 \sqrt{\lambda}} \\ \lambda(1 / \sqrt{\lambda}-|y|)^{2}, & \frac{1}{2 \sqrt{\lambda}} \leq|y| \leq \frac{1}{\sqrt{\lambda}} \\ 0, & \frac{1}{\sqrt{\lambda}} \leq \delta .\end{cases}
$$

Combining this and our calculations of $\left.C_{\lambda}^{u}\left(\chi_{K}\right)\right)(0, y)$ and $C_{4 \lambda}^{u}\left(\chi_{K}\right)(0, y)$, we obtain

$$
I_{\lambda}\left((0, y) ; K_{-}\right)=I_{\lambda}((0, y) ; K)=I_{\lambda}\left((0, y) ; K_{+}\right)=0
$$

for $|y| \leq \delta$.

The general case follows from Proposition 2.10 for the convex envelope as we may assume that $x=0 \in \mathbb{R}^{n}$ and $e=(0, \ldots, 0,1)$ and notice that in the twodimensional case, both $\chi_{K_{-}}(x, y)$ and $\chi_{K_{+}}(x, y)$ are even functions of $y$. 
Proof of Lemma 3.7. From the expression of $I_{\lambda}(x ; K)$, we have that for $x \in K$

$$
\begin{aligned}
I_{\lambda}(x ; K) & =C_{4 \lambda}^{u}\left(\chi_{K}\right)(x)-2\left(C_{\lambda}^{u}\left(\chi_{K}\right)(x)-C_{\lambda}^{l}\left(C_{\lambda}^{u}\left(\chi_{K}\right)\right)(x)\right) \\
& =2 C_{\lambda}^{l}\left(C_{\lambda}^{u}\left(\chi_{K}\right)\right)(x)+C_{4 \lambda}^{u}\left(\chi_{K}\right)(x)-2 C_{\lambda}^{u}\left(\chi_{K}\right)(x) \\
& =2 C_{\lambda}^{l}\left(C_{\lambda}^{u}\left(\chi_{K}\right)\right)(x)-1 \geq 2 C_{\lambda}^{l}\left(D_{\lambda}^{2}(\cdot ; K)\right)(x)-1
\end{aligned}
$$

where we have taken into account (2.11) and that Theorem 2.8 gives, for $x \in K$,

$$
C_{4 \lambda}^{u}\left(\chi_{K}(x)\right)-2 C_{\lambda}^{u}\left(\chi_{K}(x)\right)=-1 .
$$

The result then follows by invoking the assumption that $C_{\lambda}^{l}\left(D_{\lambda}^{2}(\cdot ; K)\right)(x)>1 / 2$.

Proof of Theorem 3.8. If $\operatorname{span}\left[e_{1}, e_{2}, \ldots, e_{m}\right]:=E \neq \mathbb{R}^{n}$, then $E$ is a proper subspace of $\mathbb{R}^{n}$. Let $e_{0} \perp E$ be a unit vector perpendicular to $E$, then $e_{0}$ is a regular direction of $0 \in L$ for any $\delta>0$. Therefore by Theorem 3.4, $C_{\lambda}^{l}\left(C_{\lambda}^{u}\left(\chi_{L}\right)\right)(0)=1 / 2$. We also have $1 / 2=C_{\lambda}^{l}\left(C_{\lambda}^{u}\left(\chi_{L}\right)\right)(0) \geq C_{\lambda}^{l}\left(D_{\lambda}^{2}(\cdot ; L)\right)(0)$. In fact we can show that $C_{\lambda}^{l}\left(D_{\lambda}^{2}(\cdot ; L)(0)=1 / 2\right.$ from the proof below for the other case.

Conversely, suppose $\operatorname{span}\left[e_{1}, e_{2}, \ldots, e_{m}\right]=\mathbb{R}^{n}$. We have, for each $i=1,2, \ldots, m$ that

$$
\operatorname{dist}\left(x ; L_{i}^{+}\right)= \begin{cases}\left|P_{e_{i}^{\perp}} x\right|, \text { if } x \cdot e_{i} \geq 0, \\ |x|, \quad \text { if } x \cdot e_{i} \geq 0 .\end{cases}
$$

Thus if we set $y=\sqrt{\lambda} x$, then

$$
\left(1-\sqrt{\lambda} \operatorname{dist}\left(x ; L_{i}^{+}\right)\right)^{2}+\lambda|x|^{2}=\left(1-\operatorname{dist}\left(y ; L_{i}^{+}\right)\right)^{2}+|y|^{2},
$$

hence

$$
D_{\lambda}^{2}(x ; L)+\lambda|x|^{2}=D_{1}^{2}(y ; L)+|y|^{2} .
$$

It follows that $C_{\lambda}^{l}\left(D_{\lambda}^{2}(\cdot ; L)\right)(0)=C_{1}^{l}\left(D_{1}^{2}(\cdot ;, L)\right)(0)$ which is independent of $\lambda>0$. Indeed, by definition, there are $\lambda_{1}>0, \ldots, \lambda_{k}>0(1 \leq k \leq n+1)$ with $\sum_{i=1}^{k} \lambda_{k}=1$ and $x_{1}, \ldots, x_{k} \in \mathbb{R}^{n}$ such that

$$
\begin{aligned}
C_{\lambda}^{l}\left(D_{\lambda}^{2}(\cdot ; L)\right)(0) & =\operatorname{co}\left[D_{\lambda}^{2}(\cdot ; L)+\lambda|x|^{2}\right](x=0)=\sum_{i=1}^{k} \lambda_{i}\left(D_{\lambda}^{2}\left(x_{i} ; L\right)+\lambda\left|x_{i}\right|^{2}\right) \\
& =\sum_{i=1}^{k} \lambda_{i}\left(D_{1}^{2}\left(y_{i} ; L\right)+\left|y_{i}\right|^{2}\right) \geq C_{1}^{l}\left(D_{1}^{2}(\cdot ; L)\right)(0),
\end{aligned}
$$

where $y_{i}=\sqrt{\lambda} x_{i}$. On the other hand, by using a similar argument we see that

$$
C_{1}^{l}\left(D_{1}^{2}(\cdot ; L)\right)(0) \geq C_{\lambda}^{l}\left(D_{\lambda}^{2}(\cdot ; L)\right)(0) .
$$

Now since $m \geq n$, we may assume, without loss of generality, that the first $n$ vectors $e_{1}, e_{2}, \ldots, e_{n}$ are linearly independent, hence form a basis of $\mathbb{R}^{n}$. Let $L^{n}=\cup_{i=1}^{n} L_{i}^{+}$. 
Then we have $D_{\lambda}^{2}(x ; L) \geq D_{\lambda}^{2}\left(x ; L^{n}\right)$ for all $x \in \mathbb{R}^{n}$ so that $C_{\lambda}^{l}\left(D_{\lambda}^{2}(\cdot ; L)\right)(x) \geq$ $C_{\lambda}^{l}\left(D_{\lambda}^{2}\left(\cdot ; L^{n}\right)\right)(x)$ for all $x \in \mathbb{R}^{n}$. Now we only need to prove that

$$
C_{1}^{l}\left(D_{1}^{2}\left(\cdot ; L^{n}\right)\right)(0)=1 / 2+\mu_{L^{n}}
$$

with $\mu_{L^{n}}>0$. We have

$$
D_{1}^{2}\left(y ; L^{n}\right)+|y|^{2}= \begin{cases}\left(1-\left|P_{e_{i}^{\perp}} y\right|\right)^{2}+|y|^{2}, & \text { if } y \in J_{i}, i=1,2, \ldots, n, \\ (1-|y|)^{2}+|y|^{2}, & \text { if } y \in J_{0} \\ |y|^{2}, & \text { if } y \in J_{+},\end{cases}
$$

where

$$
\begin{aligned}
& J_{i}=\left\{y \in \mathbb{R}^{n}, y \cdot e_{i} \geq 0,\left|P_{e_{i}^{\perp}} y\right| \leq \operatorname{dist}\left(y, L_{j}\right),\left|P_{e_{i}} y\right| \leq 1, j=1,2, \ldots, j \neq i\right\}, \\
& J_{0}=\left\{y \in \mathbb{R}^{n}, y \cdot e_{j} \leq 0, j=1,2, \ldots, n,|y| \leq 1\right\}, \\
& J_{+}=\left\{y \in \mathbb{R}^{n}, \operatorname{dist}\left(y, L^{n}\right) \geq 1\right\} .
\end{aligned}
$$

Clearly, 0 is an exposed point of the convex cone of $L^{n}$. Thus the set $K_{0}:=\{y \in$ $\left.\mathbb{R}^{n},|y|=1 / 2, y \cdot e_{i} \leq 0, i=1,2, \ldots, n\right\}=J_{0} \cap\left\{y \in \mathbb{R}^{n},|y|=\frac{1}{2}\right\}$ is not empty. We claim that

$$
\min \left\{D_{1}^{2}(y ; L)+|y|^{2}, y \in \mathbb{R}^{n}\right\}=1 / 2
$$

and the set of minimum points is exactly $K_{0}$.

By definition, in the region $J_{i}$,

$$
D_{1}^{2}(y ; L)+|y|^{2}=\left(1-\left|P_{e_{i}^{\perp}} y\right|\right)^{2}+|y|^{2}=2\left(\left|P_{e_{i}^{\perp}} y\right|-1 / 2\right)^{2}+\left|P_{e_{i}} y\right|^{2}+1 / 2 \geq 1 / 2,
$$

and the equality holds if and only if $\left|P_{e_{i}^{\perp}} y\right|=1 / 2$ and $P_{e_{i}} y=0$, which is equivalent to $y \perp e_{i}$ and $\left|P_{e_{i}^{\perp}} y\right|=|y|=1 / 2$. Since $y \in J_{i}$, by definition, $|y|=\left|P_{e_{i}^{\perp}} y\right| \leq$ $\operatorname{dist}\left(y ; L_{j}\right)$ for all $j=1,2, \ldots, n, j \neq i$. Thus $y \cdot e_{j} \leq 0$ for all $j=1,2, \ldots, n, j \neq i$. Therefore $y \in J_{0}$ and $|y|=1 / 2$ hence $y \in K_{0}$.

If $y \in J_{0}$, clearly $D_{1}^{2}(y ; L)+|y|^{2}=2(|y|-1 / 2)^{2}+1 / 2 \geq 1 / 2$ and the minimum is reached exactly when $y \in K_{0}$.

If $y \in J_{+}$, as $0 \in L^{n}, D_{1}^{2}(y ; L)+|y|^{2}=|y|^{2} \geq \operatorname{dist}^{2}\left(y ; L^{n}\right) \geq 1$. Thus both of our claims above are proved.

Clearly, $0 \notin K_{0}$. Next we show that $0 \notin \operatorname{co}\left[K_{0}\right]$ with co[$\left[K_{0}\right]$ the convex hull of $K_{0}$. If $0 \in \operatorname{co}\left[K_{0}\right]$, there are $y_{1}, \ldots, y_{k} \in K_{0}, \lambda_{1}>0, \ldots, \lambda_{k}>0$ with $1<k \leq n+1$ such that $\sum_{i=1}^{k} \lambda_{i}=1$ and $\sum_{i=1}^{k} \lambda_{i} y_{i}=0$. Now we have, for each $j=1,2, \ldots n$, $\sum_{i=1}^{k} \lambda_{i}\left(y_{i} \cdot e_{j}\right)=0$. As $y_{i} \in K_{0}$ for $i=1,2, \ldots, k$, by definition, $y_{i} \cdot e_{j} \leq 0$. Since $\lambda_{i}>0$ for all $i=1,2, \ldots, k$, we see that $y_{i} \cdot e_{j}=0$ for all $i=1,2, \ldots, k$ and $j=1,2, \ldots n$. As $e_{1}, e_{2}, \ldots, e_{n}$ is a basis of $\mathbb{R}^{n}$, we conclude that $y_{i}=0$ for all $i=1,2, \ldots, k$, which contradicts the definition of $K_{0}$.

Now we show that $C_{1}^{l}\left(D_{1}^{2}\left(\cdot ; L^{n}\right)\right)(0)>1 / 2$. This is easy to see by definition of the lower transform. Suppose $C_{1}^{l}\left(D_{1}^{2}\left(\cdot ; L^{n}\right)\right)(0)=1 / 2$, there are $y_{1}, \ldots, y_{k} \in \mathbb{R}^{n}$, 
$\lambda_{1}>0, \ldots, \lambda_{k}>0$ for some $1 \leq k \leq n+1$ with $\sum_{i=1}^{k} \lambda_{i}=1$ and $\sum_{i=1}^{k} \lambda_{i} y_{i}=0$ such that

$$
1 / 2=C_{1}^{l}\left(D_{1}^{2}\left(\cdot ; L^{n}\right)\right)(0)=\sum_{i=1}^{k} \lambda_{i}\left(D_{1}^{2}\left(y_{i} ; L^{n}\right)+\left|y_{i}\right|^{2}\right)
$$

Since $1 / 2$ is the minimum value of $D_{1}^{2}\left(y ; L^{n}\right)+|y|^{2}$, we see that $y_{i} \in K_{0}$, hence $0 \in \operatorname{co}\left[K_{0}\right]$, which is a contradiction.

Proof of Proposition 3.9. For each $\gamma_{i}:[0, \delta] \mapsto \mathbb{R}^{n}$, we may assume that the image $\Gamma_{i}$ is a graph of a function defined over the tangent line $L_{i}^{+}$, that is, there is a function $f_{i}:[0, \delta] \mapsto e_{i}^{\perp}$ such that the curve $\Gamma_{i}$ can be parameterized as $g_{i}(t)=t e_{i}+f_{i}(t)$ with $f(0)=0, f_{i}^{\prime}\left(0_{+}\right)=0$ and $\left|f_{i}^{\prime \prime}(t)\right| \leq M$ for $t \in\left[0, \delta_{i}\right]$ with $\delta_{i}>0$ a constant for $i=1,2, \ldots, m$. Let $\lambda>0$ be sufficiently large such that $R_{\lambda}:=$ $2 \sqrt{2} / \sqrt{\lambda}<\delta_{i}$ for $i=1,2, \ldots, m$. Now we define $\Gamma_{i, \lambda}=\left\{t e_{i}+f_{i}(t), 0 \leq t \leq R_{\lambda}\right\}$ and $L_{i, \lambda}^{+}=\left\{t e_{i}, 0 \leq t \leq R_{\lambda}\right\}$. Then we have, for $0 \leq t \leq R_{\lambda}$,

$$
\operatorname{dist}\left(t e_{i} ; \Gamma_{i, \lambda}\right) \leq\left|t e_{i}-\left(t e_{i}+f_{i}(t)\right)\right|=\left|f_{i}(t)\right| \leq\left|\int_{0}^{1}(1-s) f_{i}^{\prime \prime}(s t) t^{2} d s\right| \leq \frac{M R_{\lambda}^{2}}{2} .
$$

Similarly, for $0 \leq t \leq R_{\lambda}$,

$$
\operatorname{dist}\left(t e_{i}+f_{i}(t) ; L_{i, \lambda}^{+}\right) \leq\left|f_{i}(t)\right| \leq \frac{M R_{\lambda}^{2}}{2} .
$$

Thus

$$
\operatorname{dist}_{\mathcal{H}}\left(L_{i, \lambda}^{+}, \Gamma_{i, \lambda}\right) \leq \frac{M R_{\lambda}^{2}}{2} .
$$

Now we define, for each $i=1,2, \ldots, m$, the sets

$H_{i, \lambda}=\left\{t e_{i}+w, 0 \leq t \leq R_{\lambda}, w \in e_{i}^{\perp},|w| \leq \frac{M R_{\lambda}^{2}}{2}\right\} \quad$ and $\quad K_{\lambda}=\bar{B}\left(0 ; R_{\lambda}\right) \bigcup\left(\bigcup_{i=1}^{m} H_{i, \lambda}\right)$.

Note that $\Gamma_{i} \cap\left(\bar{B}\left(0 ; R_{\lambda}\right) \cup H_{i, \lambda}\right)=\Gamma_{i, \lambda}$. Now we have, by our locality property for compensated convex transforms for bounded functions, Theorem 2.6 , that

$$
\begin{aligned}
C_{\lambda}^{l} & \left(D_{\lambda}^{2}\left(\cdot ; \bigcup_{i=1}^{m} \Gamma_{i}\right)\right)(0)=C_{\lambda}^{l}\left(D_{\lambda}^{2}\left(\cdot ; \bigcup_{i=1}^{m} \Gamma_{i} \cap K_{\lambda}\right)\right)(0) \\
\quad & =C_{\lambda}^{l}\left(D_{\lambda}^{2}\left(\cdot ; \bigcup_{i=1}^{m} L_{i}^{+} \cap K_{\lambda}\right)\right)(0)+\left(C_{\lambda}^{l}\left(D_{\lambda}^{2}\left(\cdot ; \bigcup_{i=1}^{m} \Gamma_{i} \cap K_{\lambda}\right)\right)(0)-C_{\lambda}^{l}\left(D_{\lambda}^{2}\left(\cdot ; \bigcup_{i=1}^{m} L_{i}^{+} \cap K_{\lambda}\right)\right)(0)\right) \\
& :=I_{1}+I_{2} .
\end{aligned}
$$


By the locality property of lower transforms Theorem 2.6, and Theorem 3.8, we have

$$
I_{1}=C_{\lambda}^{l}\left(D_{\lambda}^{2}\left(\cdot ; \bigcup_{i=1}^{m} L_{i}^{+} \cap K_{\lambda}\right)\right)(0)=C_{\lambda}^{l}\left(D_{\lambda}^{2}\left(\cdot ; \bigcup_{i=1}^{m} L_{i}^{+}\right)\right)(0)=1 / 2+\mu_{L},
$$

whereas

$$
\begin{aligned}
\left|I_{2}\right| & =\left|C_{\lambda}^{l}\left(D_{\lambda}^{2}\left(\cdot ; \bigcup_{i=1}^{m} \Gamma_{i} \cap K_{\lambda}\right)\right)(0)-C_{\lambda}^{l}\left(D_{\lambda}^{2}\left(\cdot ; \bigcup_{i=1}^{m} L_{i}^{+} \cap K_{\lambda}\right)\right)(0)\right| \\
& \leq 2 \sqrt{\lambda} \operatorname{dist}_{\mathcal{H}}\left(\bigcup_{i=1}^{m} \Gamma_{i} \cap K_{\lambda}, \bigcup_{i=1}^{m} L_{i}^{+} \cap K_{\lambda}\right),
\end{aligned}
$$

by Lemma 2.13. By the definition of $K_{\lambda}$, we have, for each $i=1,2, \ldots, m$,

$$
\Gamma_{i} \cap K_{\lambda}=\Gamma_{i, \lambda} \bigcup\left(\bigcup_{j=1, j \neq i}^{m}\left(\left(\Gamma_{i} \cap H_{j, \lambda}\right) \backslash B\left(0 ; R_{\lambda}\right)\right)\right) \quad \text { and } \quad L_{i}^{+} \cap K_{\lambda}=L_{i, \lambda}^{+} .
$$

If $x \in \Gamma_{i, \lambda}$, we have

$$
\operatorname{dist}\left(x ; \bigcup_{i=1}^{m} L_{i}^{+} \cap K_{\lambda}\right) \leq \operatorname{dist}\left(x ; L_{i, \lambda}^{+}\right) \leq \frac{M R_{\lambda}^{2}}{2},
$$

whereas if $x \in \Gamma_{i} \cap H_{j, \lambda} \backslash B\left(0 ; R_{\lambda}\right)$, then $x=t e_{j}+w$ for some $0 \leq t \leq R_{\lambda}$ and $w \in e_{i}^{\perp}$ with $|w| \leq \frac{M R_{\lambda}^{2}}{2}$, so that

$$
\operatorname{dist}\left(x ; \bigcup_{i=1}^{m} L_{i}^{+} \cap K_{\lambda}\right) \leq\left|x-t e_{j}\right|=|w| \leq \frac{M R_{\lambda}^{2}}{2} .
$$

Thus

$$
\operatorname{dist}\left(x ; \bigcup_{i=1}^{m} L_{i}^{+} \cap K_{\lambda}\right) \leq \frac{M R_{\lambda}^{2}}{2}
$$

for all $x \in \Gamma_{i} \cap K_{\lambda}$.

Now if $y \in \bigcup_{i=1}^{m} L_{i}^{+} \bigcap K_{\lambda}$, then $x \in L_{i, \lambda}^{+}$for some $i=1,2, \ldots, m$, so that

$$
\operatorname{dist}\left(y ; \bigcup_{i=1}^{m} \Gamma_{i} \cap K_{\lambda}\right) \leq \operatorname{dist}\left(y ; \Gamma_{i, \lambda}\right) \leq \frac{M R_{\lambda}^{2}}{2} \text {. }
$$

Thus

$$
\operatorname{dist}_{\mathcal{H}}\left(\bigcup_{i=1}^{m} \Gamma_{i} \cap K_{\lambda}, \bigcup_{i=1}^{m} L_{i}^{+} \cap K_{\lambda}\right) \leq \frac{M R_{\lambda}^{2}}{2}
$$

so that

$$
\left|I_{2}\right| \leq 2 \sqrt{\lambda} \frac{M R_{\lambda}^{2}}{2}=\frac{2(2 \sqrt{2})^{2} M}{\sqrt{\lambda}} \leq \mu_{L} / 2,
$$


when $\lambda>0$ is sufficiently large. Therefore

$$
C_{\lambda}^{l}\left(D_{\lambda}^{2}\left(\cdot ; \bigcup_{i=1}^{m} \Gamma_{i}\right)\right)(0) \geq I_{1}-\left|I_{2}\right| \geq \frac{1}{2}+\frac{\mu_{L}}{2} .
$$

Consequently,

$$
I_{\lambda}\left(0 ; \bigcup_{i=1}^{m} \Gamma_{i}\right) \geq J_{\lambda}\left(0 ; \bigcup_{i=1}^{m} \Gamma_{i}\right) \geq \mu_{L}>0 .
$$

The proof is completed.

Proof of Theorem 3.10. This is a direct consequence of Theorem 3.8 and Proposition 3.9 given that

$$
C_{\lambda}^{l}\left(D_{\lambda}^{2}\left(\cdot ; \bigcup_{j=1}^{m} M_{j}\right)\right)(0) \geq C_{\lambda}^{l}\left(D_{\lambda}^{2}\left(\cdot ; \bigcup_{j=1}^{m} \Gamma_{j}\right)\right)(0)
$$

if we let $\Gamma_{j}$ be the image set of the mapping $\gamma_{i}$.

Proof of Corollary 3.11. Suppose $V_{i}:=\left\{e_{1}^{(i)}, \ldots, e_{s_{i}}^{(i)}\right\}$ is a basis of $T_{0} M_{i}$, $i=1,2, \ldots, k$, where $s_{i}=\operatorname{dim}\left(T_{0} M_{i}\right)$. Then if we consider the exponential map ${ }^{6,14,3}$ $\gamma_{i, j}(s):=\exp \left(t e_{j}^{(i)}\right), 0 \leq s \leq \delta_{i, j}$, with $\delta_{i, j}>0, i=1,2, \ldots, k, j=1,2, \ldots, s_{i}$, then $\gamma_{i, j}(s)$ are smooth geodesics of $M_{i}$. As $\operatorname{span}\left[V_{1}, \ldots, V_{k}\right]=\mathbb{R}^{n}$, the assumptions of Theorem 3.10 apply and the conclusion follows.

Proof of Corollary 3.12. Since both $M_{1}$ and $M_{2}$ are smooth, we can find linearly independent unit vectors $e_{1}^{(i)}, \ldots, e_{n-1}^{(i)} \in T_{0} M_{i}, i=1,2$ such that $g_{i, j}(s)=$ $\exp \left(s e_{j}^{(i)}\right) \in M_{i}$ are geodesics starting from 0 . This can be seen from the local charts of $M_{i}$ containing 0 . By Theorem 3.10, the conclusion then follows.

\section{Acknowledgment}

KZ wishes to thank China NSF for its partial support through grant 40771094, AO acknowledges the financial support of the Argentinean Agency through the Project Prestamo BID PICT PRH 30 No 94 and the National University of Tucumán through the project PIUNT E527, and EC is grateful for the financial support of the College of Science, Swansea University.

\section{References}

1. L. Ambrosio, P. Tilli, Topics on Analysis in Metric Spaces, (Oxford Univ. Press, London, 2004).

2. H. Attouch, D. Aze, Approximations and regularizations of arbitrary functions in Hilbert spaces by the Lasry-Lions methods, Anal. Non-Lin. H. Poincaré Inst. 10 (1993) 289-312.

3. H. Busemann, The Geometry of Geodesics, (Academic Press, New York, 1955).

4. R. Van Den Boomgaard, Mathematical Morphology: Extensions towards Computer Vision, (PhD Thesis, University of Amsterdam, 1992) 
5. R. Van den Boomgaard, A. W. M. Smelders, The morphological structure of images, IAPR International Conference on Pattern Recognition. IEEE Computer Society Press, Los Alamitos, CA (1992) 268-271.

6. J. Cheeger, D. G. Ebin, Comparison Theorems in Riemannian Geometry, (NorthHolland, 1975).

7. M. G. Crandall, H. Ishii, P.-L. Lions, User's guide to visosity solutions of second order partial differential equations, Bull. Am. Math. Soc. 27 (1992) 1-67.

8. P. Cannarsa, C. Sinestrari, Semiconcave Functions, Hamilton-Jacobi Equations and Optimal Control, (Birkhäuser, Boston, 2004).

9. J. Cao, S. Wushour, X. Yao, N. Li, J. Liang, X. Liang, Sharp feature extraction in point clouds, IET Image Process 6 (2012) 863-869.

10. K. Demarsin, D. Vanderstraeten, T. Volodine, D. Roose, Detection of closed sharp edges in point clouds using normal estimation and graph theory, Computer-Aided Design 39 (2007) 276-283.

11. J.-B. Hiriart-Urruty, C. Lemaréchal, Fundamentals of Convex Analysis, (Springer, Berlin, 2001).

12. P. T. Jackway, Morphological scale-space, IAPR International Conference on Pattern Recognition. IEEE Computer Society Press, Los Alamitos, CA (1992) 252-255.

13. P. T. Jackway, M. Deriche, Scale-space properties of the multiscale morphological dilation-erosion, IEEE Transactions on Pattern Analysis and Machine intelligence 18 (1996) 38-51

14. S. Kobayashi, K. Nomizu, Foundations of Differential Geometry, Vol. I $\&$ Vol. II, (Interscience Wiley \& Sons, New York, 1969).

15. J. M. Lasry, P. L. Lions, A remark on regularization in Hilbert Spaces, Israel Math. J. 55 (1986) 257-266.

16. Y. Lucet, What shape is your conjugate? A survey of computational convex analysis and its applications, SIAM J. Optim. 20 (2009) 216-250.

17. J.-J. Moreau, Proximaté dualité dans un espace Hilbertien, Bull. Soc. Math. Fr. 93 (1965) 273-299.

18. J.-J. Moreau, Fonctionnelles Convexes, (Lecture Notes, Collége de France, 1966).

19. N. M. Patrikalakis, T. Maekawa, Shape Interrogation for Computer Aided Design and Manufacturing, (Springer, Heidelberg, 2002).

20. J. Serra, Image Analysis and Mathematical Morphology, (Academic Press, London, 1982).

21. J. Serra, L. Vincent, An overview of morphological filtering, Circuits Systems Signals Process 11 (1992) 47-108

22. P. Soille, Morphological Image Analysis, (Springer, Berlin, 2nd Ed., 2004).

23. R. T. Rockafellar, Convex Analysis, (Princeton University Press, 1966).

24. C. Weber, S. Hahmann, H. Hagen, Sharp feature detection in point clouds, IEEE Shape Modeling International Conference (SMI), Aix-en-Provence, France (2010) 175-186.

25. K. Zhang, Compensated convexity and its applications, Anal. Non-Lin. H. Poincaré Inst. 25 (2008) 743-771.

26. K. Zhang, Convex analysis based smooth approximations of maximum functions and squared-distance functions, J. Nonlinear Convex Anal. 9 (2008) 379-406.

27. K. Zhang, A. Orlando, E.C.M. Crooks, Compensated convexity and Hausdorff stable geometric singularity extraction, Accepted for publication in M3AS Math. Models and Methods in Applied Sciences.

28. K. Zhang, E.C.M. Crooks, A. Orlando, Compensated convexity transforms and numerical algorithms, In preparation. 